\title{
An invariance principle for random planar maps
}

\author{
Grégory Miermont $\|^{1}$ \\ ${ }^{1}$ CNRS \& Laboratoire de Mathématique Equipe Probabilités, Statistique et Modélisation, Bât. 425, Université Paris- \\ Sud, 91405 Orsay, France
}

\begin{abstract}
We show a new invariance principle for the radius and other functionals of a class of conditioned 'Boltzmann-Gibbs'distributed random planar maps. It improves over the more restrictive case of bipartite maps that was discussed in Marckert and Miermont (2006). As in the latter paper, we make use of a bijection between planar maps and a class of labelled multitype trees, due to Bouttier et al. (2004). We also rely on an invariance principle for multitype spatial Galton-Watson trees, which is proved in a companion paper.
\end{abstract}

Keywords: Random planar map, invariance principle, multitype spatial Galton-Watson tree, Brownian snake

\section{Planar maps}

A planar map is a proper embedding of a connected graph in the 2-dimensional sphere, considered up to images by orientation-preserving homeomorphisms of the sphere. As an embedded graph, it has edges and vertices, and its faces are the connected components of the complementary of the embedding. The degree of a face is the number of edges that are adjacent to it, where an ithmus, i.e. an edge whose removal disconnects the graph, must be counted twice. We let $V(\mathbf{m}), E(\mathbf{m})$ and $F(\mathbf{m})$ be the sets of vertices, edges and faces of the planar map $\mathbf{m}$, while $\operatorname{deg} v, \operatorname{deg} f$ will denote the respective degrees of elements $v \in V(\mathbf{m}), f \in F(\mathbf{m})$. For any planar map $\mathbf{m}$, let $d_{\mathbf{m}}$ denote the usual graph distance on the set $V(\mathbf{m})$.

A map is pointed if a vertex (the base vertex) is distinguished, and rooted if an oriented edge (the root edge) is distinguished. The basic set of maps that we consider in this paper is the set $\mathcal{M}$ of rooted, pointed planar maps, i.e. of triples $(\mathbf{m}, r, \vec{e})$, where $\mathbf{m}$ is a planar map, $r \in V(\mathbf{m})$ is a distinguished vertex, and $\vec{e}$ is a distinguished edge. For convenience, we will usually omit the mention of $r, \vec{e}$ in the notation. For technical reasons, we assume $\mathcal{M}$ also contains the vertex map, denoted by $\dagger$, which is the planar map with no edge and only one vertex bounding a unique face of degree 0 . For $\mathbf{m} \in \mathcal{M}$, we let $\mathcal{R}(\mathbf{m})=\max _{v \in V(\mathbf{m})} d_{\mathbf{m}}(r, v)$ be the radius of the map, when seen from the base vertex. We also let $\left(\mathcal{J}^{\mathbf{m}}(k), k \geq 0\right)$ be the profile of $\mathbf{m}$, which is defined as the probability measure on $\mathbb{Z}_{+}$such that

$$
\mathcal{J}^{\mathbf{m}}(k)=\frac{\#\left\{v \in V(\mathbf{m}): d_{\mathbf{m}}(r, v)=k\right\}}{\# V(\mathbf{m})}, \quad n \geq 0 .
$$

Random planar maps have been an object of increasing interest to physicists during the last 15 years, as they can be interpreted as a discrete version of random surfaces, see Ambjørn et al. (1997) for example. It is conjectured that properly rescaled random planar maps conditioned to be large should converge to a limiting 'continuum random surface', whose law should be insensitive to (reasonable) changes in the way that the maps are sampled. The (still hypothetic) common limiting object must be of Hausdorff dimension 4, as a consequence of recent advances in Le Gall (2006). This is consistent with results of Angel (2003) and Chassaing and Durhuus (2006), who work in the different context of local limits of planar maps, i.e. when one lets the size of the map go to infinity, without performing a rescaling operation.

The first results in this direction are due to Chassaing and Schaeffer (2004), who show that typical distances in a uniform rooted quadrangulation with $n$ faces scale as $n^{1 / 4}$ when $n \rightarrow \infty$. They obtain a description of rescaled limiting radius and profile of the map, in terms of the so-called ISE (Integrated Super-Brownian Excursion). Le Gall (2006) has obtained similar results, making use of a conditioned version of the Brownian snake, which is intimately related to ISE and was introduced in Le Gall and Weill

†Gregory.Miermont@math.u-psud.fr 


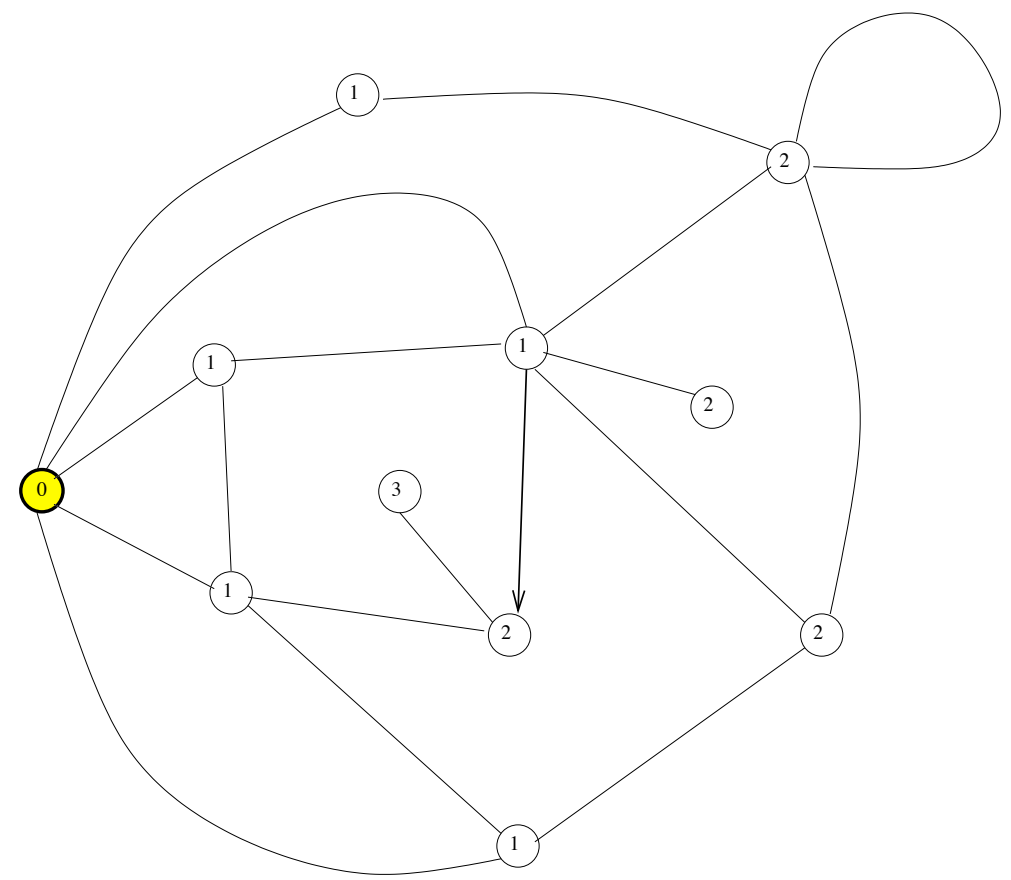

Fig. 1: A typical element of $\mathcal{M}$. It has 11 vertices, 18 edges ( 2 of which are ithmi) and 9 faces, one of degree 1 , three of degree 3 , one of degree 4 , two of degree 5 and two of degree 6 . The vertices are labelled according to their respective geodesic distances to the base vertex.

(2006). Marckert and Mokkadem (2006) show that a slightly different family of random quadrangulations with $n$ faces (pointed, and uniformly rooted on an edge adjacent to the distinguished vertex) converges in a loose sense, once rescaled by the factor $n^{1 / 4}$, to a random metric space they call the Brownian map, which is thus a conjectural limiting object for stronger topologies on (isometry classes of) metric spaces, like the Gromov-Hausdorff topology Burago et al. (2001). Marckert and Miermont (2006) show that similar results as in Chassaing and Schaeffer (2004); Le Gall (2006); Marckert and Mokkadem (2006) hold for rooted and pointed bipartite random planar maps (whose faces all have even degree) taken under a Boltzmann-type distribution, where each face of degree $2 k$ is attributed energy $\log q_{k}$, under regularity assumptions on the non-negative sequence $\left(q_{k}, k \geq 1\right)$.

The goal of the present paper is to show how results of Marckert and Miermont (2006) can be generalized to Boltzmann-type distributions on sets of maps without constraints on the degree of faces. As an important particular example, we obtain the invariance principle for the radius and profile of a uniform rooted and pointed triangulation (whose faces are all triangles) with $n$ vertices, as $n \rightarrow \infty$. Just as in Chassaing and Schaeffer (2004); Le Gall (2006); Marckert and Miermont (2006), we make use of a bijective method which encodes maps with the help of certain labelled multitype trees. This bijection is due to Bouttier et al. (2004), and generalizes the bijections of Schaeffer (1998) and Cori and Vauquelin (1981). In the case of general planar maps, the bijection of Bouttier et al. (2004) is notably more delicate to use than one encoding bipartite maps, and requires a more powerful invariance principle for multitype trees (Theorem 2), which is proved in the companion paper Miermont (2006).

The paper is organized as follows. The rest of Section 1 is devoted to the statement of our main result (Theorem 11. The key property for proving Theorem 1 is the fact that Boltzmann-distributed random maps can be conveniently encoded in spatial multitype Galton-Watson trees. Section 2 introduces the necessary formalism on Galton-Watson trees, and states the invariance principle for such trees. Then, in Section 3 . we describe the key property just mentioned, and prove Theorem 11. Finally, Section 4 discusses some extensions and perspectives for our results.

\subsection{Boltzmann distributions on maps}

Write $\mathbb{N}=\{1,2, \ldots\}$, and let $\mathbf{q}:=\left(q_{1}, q_{2}, \ldots\right) \in \mathbb{R}_{+}^{\mathbb{N}}$ be a non-negative sequence. We always assume that $q_{k}>0$ for some odd $k \geq 3$, and let $q_{0}=1$ by convention. For a planar map $\mathbf{m}$ (not necessarily pointed or 
rooted) we let

$$
W_{\mathbf{q}}(\mathbf{m})=\prod_{f \in F(\mathbf{m})} q_{\operatorname{deg} f},
$$

and assimilate $W_{\mathbf{q}}$ with the non-negative measure on $\mathcal{M}$ assigning weight $W_{\mathbf{q}}(\mathbf{m})$ to $\{(\mathbf{m}, r, \vec{e})\}$. We say that $\mathbf{q}$ is admissible if the 'partition function' $Z_{\mathbf{q}}:=W_{\mathbf{q}}(\mathcal{M})$ is finite. It always hold that $Z_{\mathbf{q}}>1$ because $W_{\mathbf{q}}(\{\dagger\})=1$ and by the hypothesis that $q_{k}>0$ for at least one $k>1$. Hence, if $\mathbf{q}$ is admissible, the expression

$$
P_{\mathbf{q}}=\frac{W_{\mathbf{q}}}{Z_{\mathbf{q}}},
$$

defines a Boltzmann-type probability distribution on $\mathcal{M}$. We let $M: \mathcal{M} \rightarrow \mathcal{M}$ be the identity mapping.

It is interesting to note that a random variable with distribution $P_{\mathbf{q}}$ can be obtained in the following way: choose the rooted map $(\mathbf{m}, \vec{e})$ with probability proportional to $W_{\mathbf{q}}(\mathbf{m})$, and given this choice, choose the base vertex uniformly at random among the $\# V(\mathbf{m})$ possible. This observation comes from the fact that a root-preserving automorphism of a rooted planar map has to be the identity, so that each choice of a base vertex in a given rooted planar map yields a distinct element of $\mathcal{M}$.

For the needs of our study, we will have to introduce the three subsets of $\mathcal{M}$ defined as follows. For $(\mathbf{m}, r, \vec{e}) \in \mathcal{M}$, we let $e_{-}, e_{+}$be the source and target vertices of the edge $\vec{e}$. As in Figure 1 , we label the vertices according to their distance to the base vertex, and distinguish the three possibilities

- $\mathcal{M}_{+}=\left\{(\mathbf{m}, r, \vec{e}) \in \mathcal{M}: d\left(r, e_{+}\right)=d\left(r, e_{-}\right)+1\right\} \cup\{\dagger\}$,

- $\mathcal{M}_{-}=\left\{(\mathbf{m}, r, \vec{e}) \in \mathcal{M}: d\left(r, e_{+}\right)=d\left(r, e_{-}\right)-1\right\}$,

- $\mathcal{M}_{0}=\left\{(\mathbf{m}, r, \vec{e}) \in \mathcal{M}: d\left(r, e_{+}\right)=d\left(r, e_{-}\right)\right\}$.

On Figure 1 is an element of $\mathcal{M}_{+}$. Notice that $\mathcal{M}_{-}$does not contain the vertex map $\dagger$, which is the convention we need so that $\mathcal{M}$ is partitioned into $\mathcal{M}_{+}, \mathcal{M}_{-}, \mathcal{M}_{0}$. The elements of $\mathcal{M}_{+} \backslash\{\dagger\}$ and $\mathcal{M}_{-}$are in a natural one-to-one correspondence (reverse the orientation of $\vec{e}$ ). We define

$$
Z_{\mathbf{q}}^{+}=W_{\mathbf{q}}\left(\mathcal{M}_{+}\right), \quad Z_{\mathbf{q}}^{-}=W_{\mathbf{q}}\left(\mathcal{M}_{-}\right), \quad Z_{\mathbf{q}}^{0}=W_{\mathbf{q}}\left(\mathcal{M}_{0}\right)
$$

so by our previous observation $Z_{\mathbf{q}}^{-}=Z_{\mathbf{q}}^{+}-1$. We then have $Z_{\mathbf{q}}=Z_{\mathbf{q}}^{+}+Z_{\mathbf{q}}^{-}+Z_{\mathbf{q}}^{0}=2 Z_{\mathbf{q}}^{+}-1+Z_{\mathbf{q}}^{0}$. For admissible q, let

$$
P_{\mathbf{q}}^{+}=P_{\mathbf{q}}\left(\cdot \mid \mathcal{M}_{+}\right)=W_{\mathbf{q}}\left(\cdot \cap \mathcal{M}_{+}\right) / Z_{\mathbf{q}}^{+},
$$

and define similarly the conditioned probabilities $P_{\mathbf{q}}^{-}, P_{\mathbf{q}}^{0}$.

We define

$$
N_{\bullet}\left(k, k^{\prime}\right)=\left(\begin{array}{c}
2 k+k^{\prime}+1 \\
k+1
\end{array}\right), \quad N_{\diamond}\left(k, k^{\prime}\right)=\left(\begin{array}{c}
2 k+k^{\prime} \\
k
\end{array}\right), \quad k, k^{\prime} \in \mathbb{Z}_{+},
$$

and let, for $x, y \geq 0$,

$$
\begin{aligned}
& f_{\mathbf{q}}^{\bullet}(x, y)=\sum_{k, k^{\prime} \geq 0} x^{k} y^{k^{\prime}} N_{\bullet}\left(k, k^{\prime}\right)\left(\begin{array}{c}
k+k^{\prime} \\
k
\end{array}\right) q_{2+2 k+k^{\prime}} \\
& f_{\mathbf{q}}^{\diamond}(x, y)=\sum_{k, k^{\prime} \geq 0} x^{k} y^{k^{\prime}} N_{\diamond}\left(k, k^{\prime}\right)\left(\begin{array}{c}
k+k^{\prime} \\
k
\end{array}\right) q_{1+2 k+k^{\prime}},
\end{aligned}
$$

which both may be finite or infinite.

Proposition 1 The weight sequence $\mathbf{q}$ is admissible if and only if the system

$$
\begin{aligned}
\frac{z^{+}-1}{z^{+}} & =f_{\mathbf{q}}^{\bullet}\left(z^{+}, z^{\diamond}\right) \\
z^{\diamond} & =f_{\mathbf{q}}^{\diamond}\left(z^{+}, z^{\diamond}\right)
\end{aligned}
$$

has a solution $\left(z^{+}, z^{\diamond}\right) \in(0, \infty)^{2}$, for which the matrix

$$
\mathfrak{M}_{\mathbf{q}}\left(z^{+}, z^{\diamond}\right):=\left(\begin{array}{ccc}
0 & 0 & z^{+}-1 \\
\frac{z^{+}}{z^{\diamond}} \partial_{x} f_{\mathbf{q}}^{\diamond}\left(z^{+}, z^{\diamond}\right) & \partial_{y} f_{\mathbf{q}}^{\diamond}\left(z^{+}, z^{\diamond}\right) & 0 \\
\frac{\left(z^{+}\right)^{2}}{z^{+}-1} \partial_{x} f_{\mathbf{q}}^{\bullet}\left(z^{+}, z^{\diamond}\right) & \frac{z^{+} z^{\diamond}}{z^{+}-1} \partial_{y} f_{\mathbf{q}}^{\bullet}\left(z^{+}, z^{\diamond}\right) & 0
\end{array}\right)
$$


has spectral radius $\varrho \leq 1$. This 'good' solution is then unique and $Z_{\mathbf{q}}^{+}=z^{+}, Z_{\mathbf{q}}^{0}=\left(z^{\diamond}\right)^{2}$, so that $Z_{\mathbf{q}}=2 z^{+}-1+\left(z^{\diamond}\right)^{2}$.

Proposition 1 will be proved in Section 3.2. Notice that $Z_{\mathbf{q}}^{+}$has to be $>1$ by 4 , which is consistent with the fact $\dagger \in \mathcal{M}_{+}$. We adopt the notation $Z_{\mathbf{q}}^{\diamond}=z^{\diamond}$ whenever $\left(z^{+}, z^{\diamond}\right)$ is the good solution of (4) corresponding to an admissible $\mathbf{q}$.

Definition 1 We say that $\mathbf{q}$ is critical if it is admissible and if the matrix $\mathfrak{M}_{\mathbf{q}}:=\mathfrak{M}_{\mathbf{q}}\left(Z_{\mathbf{q}}^{+}, Z_{\mathbf{q}}^{\diamond}\right)$ has spectral radius $\varrho=1$, which amounts to the condition

$$
\left(Z_{\mathbf{q}}^{+}\right)^{2} J_{f}\left(Z_{\mathbf{q}}^{+}, Z_{\mathbf{q}}^{\diamond}\right)+1=\left(Z_{\mathbf{q}}^{+}\right)^{2} \partial_{x} f_{\mathbf{q}}^{\bullet}\left(Z_{\mathbf{q}}^{+}, Z_{\mathbf{q}}^{\diamond}\right)+\partial_{y} f_{\mathbf{q}}^{\diamond}\left(Z_{\mathbf{q}}^{+}, Z_{\mathbf{q}}^{\diamond}\right)
$$

where $J_{f}$ is the (signed) Jacobian of the function $\left(f_{\mathbf{q}}^{\bullet}, f_{\mathbf{q}}^{\diamond}\right): \mathbb{R}_{+}^{2} \rightarrow\left(\mathbb{R}_{+} \cup\{\infty\}\right)^{2}$.

We say that $\mathbf{q}$ is regular critical if it is critical and if $f_{\mathbf{q}}^{\bullet}\left(Z_{\mathbf{q}}^{+}+\varepsilon, Z_{\mathbf{q}}^{\diamond}+\varepsilon\right)<\infty$ for some $\varepsilon>0$.

Notice that we could take $f_{\mathbf{q}}^{\diamond}$ as well in the definition of regular criticality, since it holds that $\partial_{x}\left(x f_{\bullet}\right)=$ $\partial_{y} f_{\diamond}$, as can be checked from the definitions of $N_{\bullet}, N_{\diamond}$.

\subsection{Main result}

We now briefly describe the process known as the head of the Brownian snake, see Marckert and Mokkadem (2003). Let $B^{\text {ex }}$ be a standard Brownian excursion, and given $B^{\text {ex }}$, let $R^{\text {ex }}$ be a centered Gaussian process whose covariance function is given by

$$
\operatorname{Cov}\left(R_{s}^{\mathrm{ex}}, R_{t}^{\mathrm{ex}}\right)=\inf _{s \wedge t \leq u \leq s \vee t} B_{u}^{\mathrm{ex}} \quad 0 \leq s, t \leq 1 .
$$

It is well-known, see e.g. (Le Gall, 1999. Sect. IV.6), that $\left(B^{\mathrm{ex}}, R^{\mathrm{ex}}\right)$ has a continuous version, which is the one we always work with.

Our main result result generalizes (Marckert and Miermont, 2006, Proposition 4). It shows that invariance principles hold for the radius, distance to a uniformly sampled vertex, and profile of a Boltzmann-distributed critical random map, where the limiting distribution can be described in terms of the head of the Brownian snake. These distributions are, as expected, exactly the same as in the bipartite case, the only change coming from the scaling constants. In the following statement, for $\mathbf{m} \in \mathcal{M}$ and $n \geq 1$, we let $\mathcal{J}_{n}^{\mathbf{m}}$ be the measure on $\mathbb{R}_{+}$defined by $\mathcal{J}_{n}^{\mathbf{m}}(A)=\mathcal{J}^{\mathbf{m}}\left(n^{1 / 4} A\right), A$ being a Borel subset of $\mathbb{R}_{+}$.

Theorem 1 Let $\mathbf{q}$ be a regular critical weight sequence. Then, there exists a scaling constant $C_{\mathbf{q}} \in(0, \infty)$ such that

(i) The law of $n^{-1 / 4} \mathcal{R}(M)$ under $P_{\mathbf{q}}(\cdot \mid \# V(M)=n)$ converges weakly as $n \rightarrow \infty$ to the law of $C_{q}\left(\sup R^{\mathrm{ex}}-\inf R^{\mathrm{ex}}\right)$.

(ii) The law of $n^{-1 / 4} d_{M}\left(r, r^{\prime}\right)$ under $P_{\mathbf{q}}(\cdot \mid \# V(M)=n)$, where $r^{\prime}$ is a vertex picked uniformly at random in $V(M) \backslash\{r\}$ conditionally on $M$, converges weakly as $n \rightarrow \infty$ to the law of $C_{q} \sup R^{\text {ex }}$.

(iii) The distribution of the random measure $\mathrm{J}_{n}^{M}$ under $P_{\mathbf{q}}(\cdot \mid \# V(M)=n)$ converges weakly as $n \rightarrow \infty$ to the law of the random probability measure $\mathcal{J}^{\mathrm{ex}}$ on $\mathbb{R}_{+}$, defined by

$$
\operatorname{J}^{\mathrm{ex}}(g)=\int_{0}^{1} \mathrm{~d} s g\left(C_{\mathbf{q}}\left(R_{s}^{\mathrm{ex}}-\inf R^{\mathrm{ex}}\right)\right),
$$

for every non-negative measurable $g$.

In statements (i), (ii), (iii), it must be understood that $n \rightarrow \infty$ really denotes if necessary a subsequence $n_{m}, m \geq 1$ along which $P_{\mathbf{q}}\left(\# V(M)=n_{m}\right)>0$.

The strategy of our proof will be to show that Theorem 1 holds true (with the same scaling constant $C_{\mathbf{q}}$ ) when replacing $P_{\mathbf{q}}$ everywhere by $P_{\mathbf{q}}^{+}$, resp. $P_{\mathbf{q}}^{-}, P_{\mathbf{q}}^{0}$. Since these three probability measures are the conditioned versions of $P_{\mathbf{q}}$ on events that partition $\mathcal{M}$, if the limit in distribution is the same in the three cases, then in particular the convergence in distribution will also hold for $P_{\mathbf{q}}$. Of course, proving the result for $P_{\mathbf{q}}^{-}$is the same as for $P_{\mathbf{q}}^{+}$by the natural corespondence that exists between the two probabilities: for $n \geq 1, P_{\mathbf{q}}^{-}(\cdot \mid \# V(M)=n)$ is the push-forward of $P_{\mathbf{q}}^{+}(\cdot \mid \# V(M)=n)$ by the map that reverses the orientation of the root edge. As we will see in Section 3.1 , the study of $P_{\mathbf{q}}^{+}$and $P_{\mathbf{q}}^{0}$ involves two related bijections with certain sets of labeled trees. The one for $P_{\mathbf{q}}^{0}$ is trickier, but somehow without more conceptual difficulties, so we will be more sketchy on this side and essentially focus on proving Theorem 1 for $P_{\mathbf{q}}^{+}$instead of $P_{\mathbf{q}}$. 
Remarks. - In principle, the constant $C_{\mathbf{q}}$ can be computed explicitely (see Section 3.4 below), but it seems that, unlike in Marckert and Miermont (2006), there is no simple closed form for it in terms of $Z_{\mathbf{q}}^{+}, Z_{\mathbf{q}}^{\diamond}, f_{\mathbf{q}}^{\bullet}, f_{\mathbf{q}}^{\diamond}$ and their derivatives (see also the second remark in Section 1.3 .

- It should be noted that when $q_{k}=0$ for every odd $k$, which we excluded from our study, the formula (7) degenerates to the criticality condition $\left(Z_{\mathbf{q}}^{+}\right)^{2} f_{\mathbf{q}}^{\prime}\left(Z_{\mathbf{q}}^{+}\right)=1$, where $f_{\mathbf{q}}(x)=f_{\mathbf{q}}^{\bullet}(x, 0)$. This is the formula given in Marckert and Miermont (2006), and amounts to say that $Z_{\mathbf{q}}^{\diamond}$ degenerates to 0 in the case where $W_{\mathbf{q}}$ is supported by bipartite maps. Thus, the statements of Proposition 1 and Theorem 1 remain true in this case (still assuming $q_{k}>0$ for at least one $k>3$ ), provided the entries in the second row of $\mathfrak{M}_{\mathbf{q}}$ are all 0 .

- Notice that we have given a statement with a conditioning on the number of vertices of the random map under $P_{\mathbf{q}}$, while Marckert and Miermont (2006) gave results also for a conditioning with respect to the number of faces. Although we expect the result to be true (with different scaling constants) when conditioning on the number of faces, we do not have a proof for this statement. Indeed, in the bijection between maps and multitype trees that is introduced in Section 3.1. the faces of planar maps are matched with vertices of the trees of two distinct types, while vertices of the map are matched to vertices of the trees of a single third type. One the other hand, our main tool on multitype trees (Theorem 2), involves only conditionings with respect to the number of vertices of some fixed type.

However, in the particularly interesting models of Boltzmann-distributed random $k$-angulations for which all faces are of degree $k \geq 3$, conditioning on the number of vertices or faces amounts to the same, as we will now see in the particular case $k=3$.

\subsection{The example of triangulations}

A triangulation is a planar map whose faces all have degree 3 . We do not assume extra connectivity properties on the planar map such as 2 or 3-connectedness, so that it may contain loops and/or multiple edges (such maps are called type-I triangulations in Ambjørn et al. (1997)). As observed in the previous remark, conditioning a random triangulation with respect to the number of its vertices or of its faces amounts to the same, because of the linear relation $\# F(\mathbf{m})=2 \# V(\mathbf{m})-4$ satisfied by any triangulation $\mathbf{m} \neq \dagger$. This is clear from Euler's formula $\# V(\mathbf{m})-\# E(\mathbf{m})+\# F(\mathbf{m})=2$, and the formula $3 \# F(\mathbf{m})=2 \# E(\mathbf{m})$ which comes from the fact that all faces are triangles.

Let $q_{3}=q>0$ and $q_{i}=0$ for $i \in \mathbb{N} \backslash\{3\}$. In that case, $W_{\mathbf{q}}$ puts a weight $q^{n}$ on all triangulations of $\mathcal{M}$ with $n$ triangles. In particular, by the previous paragraph, the probability distribution $P_{\mathbf{q}}(\cdot \mid \# V(M)=n)$ as it appears in Theorem 1 , is the uniform distribution on the set of triangulations with $n$ vertices $(2 n-4$ triangles).

Let us look for the value $q_{\mathrm{cr}}$ of $q$ that makes $\mathbf{q}$ critical (in fact, regular critical). The functions $f_{\mathbf{q}}^{\bullet}, f_{\mathbf{q}}^{\diamond}$ are given by

$$
f_{\mathbf{q}}^{\bullet}(x, y)=2 q y, \quad f_{\mathbf{q}}^{\diamond}(x, y)=2 q x+q y^{2} .
$$

It is easy to see that 4 p amounts to $2 q Z_{\mathbf{q}}^{\diamond}=1-\sqrt{1-8 q^{2} Z_{\mathbf{q}}^{+}}=1-\left(Z_{\mathbf{q}}^{+}\right)^{-1}$, which imposes $8 q^{2} Z_{\mathbf{q}}^{+} \leq 1$. The criticality condition $(7)$ then yields $-4\left(q_{\mathrm{cr}}\right)^{2}\left(Z_{\mathbf{q}}^{+}\right)^{2}=2 q_{\mathrm{cr}} Z_{\mathbf{q}}^{\diamond}-1=-\left(Z_{\mathbf{q}}^{+}\right)^{-1}$, so that $4\left(q_{\mathrm{cr}}\right)^{2}\left(Z_{\mathbf{q}}^{+}\right)^{3}=$ 1. From this, we find

$$
Z_{\mathbf{q}}^{+}=\sqrt{3} \quad \text { and } \quad q_{\mathrm{cr}}=\frac{1}{2 \cdot 3^{3 / 4}} .
$$

We can compute the scaling constant $C_{\mathbf{q}}$ appearing in the statement of Theorem 1 as

$$
C_{q_{\mathrm{cr}} \delta_{3}}=\left(\frac{1}{3}\right)^{1 / 4}=0.75983 \ldots
$$

This will be shown in Section 3.4.2. Note that this constant is strictly less than the scaling constant $(8 / 9)^{1 / 4}$ which appears for quadrangulations.

Remarks. - Triangulations that are two or three-connected can be studied via other kinds of bijective methods, see Poulalhon and Schaeffer (2003); Poulalhon (2002) for example. It seems that it would be harder to obtain limiting laws e.g. for the radius of such constrained triangulations with the help of labeled trees as in the present paper, as it would involve intricate conditionings, see Remark 3. in Section 4

- It would be natural to try and compute the critical weight $q_{\mathrm{cr}}$, the partition function $Z_{q_{\mathrm{cr}} \delta_{k}}$ and the scaling constant $C_{q_{\mathrm{cr}} \delta_{k}}$ in the case of Boltzmann laws on $k$-angulations, where $q_{i}=q \delta_{i k}$ for some odd number $k \geq 5$. For even $k$, the scaling constant was shown to be $(k(k-2) / 9)^{1 / 4}$ in Marckert and Miermont (2006), and the formula is still valid for triangulations. A natural conjecture is that this formula is valid for all $k$. However, by contrast with the bipartite case, the complexity of computations (at least with our 
method) grows with $k$. It is already a very tedious exercise to solve (4) and (7) and find the scaling constant $C_{\mathbf{q}}$ in the case $k=3$, and the computations become yet much harder for $k=5$. Since the equations to be solved involve polynomials whose degrees increase with $k$, it might even be that there is no explicit expression for $q_{\mathrm{cr}}, Z_{q_{\mathrm{cr}} \delta_{k}}$ involving only radicals and rational fractions of $k$.

\section{Multitype spatial Galton-Watson trees}

\subsection{Multitype trees}

Let $\mathcal{U}$ be the set of finite words with alphabet $\mathbb{N}$,

$$
\mathcal{U}=\bigsqcup_{n \geq 0} \mathbb{N}^{n}
$$

where $\mathbb{N}^{n}$ is the set of words with $n$ integer letters, and by convention $\mathbb{N}^{0}=\{\varnothing\}$. For two words $u, v$, we let $u v$ be their concatenation and $|u|,|v|$ their length (with the convention $|\varnothing|=0$ ). If $u$ is a word and $A \subseteq \mathcal{U}$, we also let $u A=\{u v: v \in A\}$, and say that $u$ is a prefix of $v$ if $v \in u \mathcal{U}$. A planar tree is a finite subset $\mathbf{t}$ of $\mathcal{U}$ such that

- $\varnothing \in \mathbf{t}$, it is called the root of $\mathbf{t}$,

- for every $u \in \mathcal{U}$ and $i \in \mathbb{N}$, if $u i \in \mathbf{t}$ then $u \in \mathbf{t}$, and $u j \in \mathbf{t}$ for every $1 \leq j \leq i$.

We let $\mathcal{T}$ be the set of planar trees. For a tree $\mathbf{t} \in \mathcal{T}$ and $u \in \mathbf{t}$, the number $c^{\mathbf{t}}(u)=\max \left\{i \in \mathbb{Z}_{+}: u i \in \mathbf{t}\right\}$, with the convention $u 0=u$, is the number of children of $u$. An element $u \in \mathbf{t}$ is called a vertex of $\mathbf{t}$, and the length $|u|$ of the word $u$ is called the height of $u$ in $\mathbf{t}$. Any planar tree $\mathbf{t}$ is endowed with the linear order which is the restriction on $\mathbf{t}$ of the usual lexicographical order $\prec$ on $\mathcal{U}$ ( $u \prec v$ if $u$ is a proper prefix of $v$ or if $u=w u^{\prime}, v=w v^{\prime}$ for some $w, u^{\prime}, v^{\prime} \in \mathcal{U}$, where the first letters $u_{1}^{\prime}, v_{1}^{\prime}$ of $u^{\prime}, v^{\prime}$ satisfy $u_{1}^{\prime}<v_{1}^{\prime}$ ).

Let $K \in \mathbb{N}$ and $[K]=\{1,2, \ldots, K\}$. A $K$-type tree is a pair $\left(\mathbf{t}, e_{\mathbf{t}}\right)$ where $e_{\mathbf{t}}: \mathbf{t} \rightarrow[K]$ assigns a type to each vertex. We let $\mathcal{T}^{(K)}$ be the set of $K$-type trees. A $K$-type spatial tree is a triple $(\mathbf{t}, e, \ell)$ where $(\mathbf{t}, e) \in \mathcal{T}^{(K)}$ and $\boldsymbol{\ell}=\left(\ell_{u}: u \in \mathbf{t}\right) \in \mathbb{R}^{\mathbf{t}}$. We let $\mathbb{T}^{(K)}$ be the set of $K$-type spatial trees. For $u \in \mathbf{t}, \ell_{u}$ can be interpreted as the position of $u$ in a 1-dimensional embedding of $\mathbf{t}$. Notice that a spatial motion $\ell$ on some tree $\mathbf{t}$ can alternatively be described by the label increments $\mathbf{y}=\left(y_{u}, u \in \mathbf{t}\right)$, where $y_{\varnothing}=\ell_{\varnothing}$, and for $u \in \mathbf{t}$ and $1 \leq i \leq c^{\mathbf{t}}(u), y_{u i}=\ell_{u i}-\ell_{u}$. The function $\ell$ is recovered by

$$
\ell_{u}=\sum_{k=1}^{n} y_{u_{1} \ldots u_{k}}, \quad u=u_{1} \ldots u_{n} \in \mathbf{t} .
$$

This notation $\mathbf{y}$ will be used frequently in this paper.

In the sequel, we will often write $e$ instead of $e_{\mathbf{t}}$ and denote elements of $\mathcal{T}^{(K)}, \mathbb{T}^{(K)}$ by $\mathbf{t}$, without explicitly mentioning the type and spatial embedding, when this is free of ambiguity. We let, for $i \in[K]$,

$$
\mathcal{T}_{i}^{(K)}=\left\{\mathbf{t} \in \mathcal{T}^{(K)}: e(\varnothing)=i\right\}
$$

and $\mathbb{T}_{i}^{(K)}=\left\{\mathbf{t} \in \mathbb{T}^{(K)}: e(\varnothing)=i\right\}$. Also, for $\mathbf{t} \in \mathcal{T}^{(K)}$ and $i \in[K]$ we will denote by $\mathbf{t}^{(i)}=e^{-1}(\{i\})$ the set of vertices of $\mathbf{t}$ with type $i$.

Finally, let $\mathcal{W}_{K}=\bigsqcup_{n>0}[K]^{n}$ be the set of finite $[K]$-valued sequences, and consider the natural projection $p: \mathcal{W}_{K} \rightarrow \mathbb{Z}_{+}^{K}$, where $p(\mathbf{w})=\left(p_{i}(\mathbf{w}), i \in[K]\right)$ and $p_{i}(\mathbf{w})=\#\left\{j: \mathbf{w}_{j}=i\right\}$. We then have $|\mathbf{w}|=|p(\mathbf{w})|_{1}$ where $|\mathbf{w}|$ is the length of $\mathbf{w}$ and $|\cdot|_{1}$ is the $l_{1}$-norm. For each $\mathbf{z} \in \mathbb{Z}_{+}^{K}$, there are exactly

$$
\#\left\{\mathbf{w} \in \mathcal{W}_{K}: p(\mathbf{w})=\mathbf{z}\right\}=\left(\begin{array}{c}
|\mathbf{z}|_{1} \\
z_{1}, \ldots, z_{K}
\end{array}\right)=\frac{\left(z_{1}+\ldots+z_{K}\right) !}{z_{1} ! \ldots z_{K} !}
$$

preimages of $\mathbf{z}$ by the mapping $p$. For $\mathbf{t} \in \mathcal{T}^{(K)}$, every $u \in \mathbf{t}$ determines a sequence

$$
\mathbf{w}_{\mathbf{t}}(u)=\left(w_{i}^{\mathbf{t}}(u), 1 \leq i \leq c^{\mathbf{t}}(u)\right)=\left(e(u i), 1 \leq i \leq c^{\mathbf{t}}(u)\right) \in \mathcal{W}_{K}
$$

with length $\left|\mathbf{w}^{\mathbf{t}}(u)\right|=c^{\mathbf{t}}(u)$. The vector $\mathbf{z}^{\mathbf{t}}(u)=p\left(\mathbf{w}^{\mathbf{t}}(u)\right)=\left(z_{i}^{\mathbf{t}}(u), 1 \leq i \leq K\right)$ counts the number of children of $u$ of each type. 


\subsection{Multitype spatial Galton-Watson trees}

\subsubsection{Galton-Watson trees}

Let now $\boldsymbol{\zeta}=\left(\zeta^{(i)}, i \in[K]\right)$ be a family of probability measures on the set $\mathcal{W}_{K}$, and let $\boldsymbol{\mu}=\left(\mu^{(i)}=\right.$ $\left.p_{*} \zeta^{(i)}, i \in[K]\right)$ be their push-forward by $p$, so that they define laws on $\mathbb{Z}_{+}^{K}$. We call $\zeta$ an ordered offspring distribution and $\boldsymbol{\mu}$ the associated unordered offspring distribution. Notice that we might always write $\zeta^{(i)}(\mathbf{w})=\mu^{(i)}(p(\mathbf{w})) \operatorname{ord}_{p(\mathbf{w})}^{(i)}(\mathbf{w})$, where for every $i \in[K], \mathbf{z} \in \mathbb{Z}_{+}^{K}, \operatorname{ord}_{\mathbf{z}}^{(i)}(\cdot)$ is a probability measure on $p^{-1}(\mathbf{z})$, which can be interpreted as a way of randomly ordering a multiset made of $z_{j}$ copies of the element $j$ for $1 \leq j \leq K$. When $\operatorname{ord}_{\mathbf{z}}^{(i)}(\cdot)$ is the uniform distribution on $p^{-1}(\mathbf{z})$, we say that $\zeta^{(i)}$ is the uniform ordering of $\mu^{(i)}$.

We make the basic assumption that

$$
\max _{i \in[K]} \mu^{(i)}\left(\left\{\mathbf{z} \in \mathbb{Z}_{+}^{K}: \sum_{j} z_{j} \neq 1\right\}\right)>0,
$$

and say that $\boldsymbol{\zeta}$, or $\boldsymbol{\mu}$, is non-degenerate. For $i, j \in[K]$, let

$$
m_{i j}=\sum_{\mathbf{z} \in \mathbb{Z}_{+}^{K}} z_{j} \mu^{(i)}(\{\mathbf{z}\})
$$

be the mean number of type- $j$ offspring of an type- $i$ individual, and let $\mathfrak{M}_{\boldsymbol{\mu}}=\left(m_{i j}\right)_{i, j \in[K]}$ and call it the mean matrix of $\boldsymbol{\mu}$. It is said to be irreducible, if for every $i, j \in[K]$, there is some $n \in \mathbb{N}$ so that $m_{i j}^{(n)}>0$, where $m_{i j}^{(n)}$ is the $i j$-entry of the matrix $\mathfrak{M}_{\boldsymbol{\mu}}^{n}$. We say that $\boldsymbol{\mu}$ is irreducible if $\mathfrak{M}_{\boldsymbol{\mu}}$ is.

Under the irreducibility assumption, the Perron-Frobenius theorem ensures that $\mathfrak{M}_{\mu}$ has a real, positive eigenvalue $\varrho$ with maximal modulus. The distribution $\zeta$ ( or $\boldsymbol{\mu}$ ), is called sub-critical if $\varrho<1$ and critical if $\varrho=1$.

Assuming $\zeta$ non-degenerate, irreducible and (sub-)critical, for every $i \in[K]$, one can construct a distribution $P_{\zeta}^{(i)}$ (or $P^{(i)}$ when free of ambiguity) on $\mathcal{T}_{i}^{(K)}$ such that

- the root $\varnothing$ has type $i$ a.s.,

- different vertices have independent sets of children, such that

- type- $j$ vertices have a set of children with types given by a sequence $\mathbf{w} \in \mathcal{W}_{K}$ with probability $\zeta^{(j)}(\mathbf{w})$.

Details of the construction of this distribution, are given in Miermont (2006). It is the law of a $K$-type Galton-Watson (GW) tree with ancestor of type $i$ and offspring distributions $\zeta$. The probability measure $P^{(i)}$ is entirely characterized by the formulae

$$
P^{(i)}\left(\left(T, e_{T}\right)=(\mathbf{t}, e)\right)=\prod_{u \in \mathbf{t}} \zeta^{(e(u))}\left(\mathbf{w}_{\mathbf{t}}(u)\right),
$$

where $\left(T, e_{T}\right): \mathcal{T}^{(K)} \rightarrow \mathcal{T}^{(K)}$ is the identity mapping and $\mathbf{t}$ ranges over $K$-type trees.

\subsubsection{Spatial motion}

Next, we couple the trees with a branching spatial motion, as follows. Consider a family $\boldsymbol{\nu}=\left(\nu_{i, \mathbf{w}}, i \in\right.$ $\left.[K], \mathbf{w} \in \mathcal{W}_{K}\right)$, where $\nu_{i, \mathbf{w}}(\mathrm{d} \mathbf{y})$ is a probability distribution on $\mathbb{R}^{|\mathbf{w}|}$.

For $(\mathbf{t}, e) \in \mathcal{T}^{(K)}$ and every $u \in \mathbf{t}$ with type $e(u)=i$ and children vector $\mathbf{w}^{\mathbf{t}}(u)=\mathbf{w}$, we take a random variable $\left(\widetilde{Y}_{u j}, 1 \leq j \leq|\mathbf{w}|\right)$ with law $\nu_{i, \mathbf{w}}$, independently over distinct $u$ 's. We use these as increments of a spatial motion on $\mathbf{t}$, that is, we set $\widetilde{L}(\varnothing)=0$ and let

$$
\widetilde{L}_{u}=\sum_{k=0}^{n} \widetilde{Y}_{u_{1} \ldots u_{k}} \quad u=u_{1} \ldots u_{n} \in \mathbf{t} .
$$

Let $\nu_{\mathbf{t}, e}$ be the law of the random vector $\left(\widetilde{L}_{u}, u \in \mathbf{t}\right)$ thus obtained. We let $\mathbb{P}_{\boldsymbol{\zeta}, \boldsymbol{\nu}}^{(i)}$ (or simply $\mathbb{P}^{(i)}$ ) be the probability measure $\mathrm{d} P_{\zeta}^{(i)}(\mathbf{t}, e) \mathrm{d} \nu_{\mathbf{t}, e}(\ell)$ defined on $\mathbb{T}_{i}^{(K)}$. Such probability measures are called laws of spatial Galton-Watson (SGW) trees. They are characterized by the formulae

$$
\mathbb{P}_{\boldsymbol{\zeta}, \boldsymbol{\nu}}^{(i)}\left(\left(T, e_{T}\right)=(\mathbf{t}, e), L \in \mathrm{d} \boldsymbol{\ell}\right)=P_{\boldsymbol{\zeta}}^{(i)}(\{\mathbf{t}, e\}) \prod_{u \in \mathbf{t}} \nu_{e(u), \mathbf{w}^{\mathbf{t}}(u)}\left(\mathrm{d}\left(y_{u j}, 1 \leq j \leq c^{\mathbf{t}}(u)\right)\right),
$$


where $(\mathbf{t}, e) \in \mathcal{T}^{(K)},\left(T, e_{T}, L\right): \mathbb{T}^{(K)} \rightarrow \mathbb{T}^{(K)}$ is the identity mapping. It should be kept in mind that the distributions $\nu_{i, \mathbf{w}}$ for which $|\mathbf{w}|=0$ or $\zeta^{(i)}(\mathbf{w})=0$ are irrelevant in the definition of $\mathbb{P}_{\boldsymbol{\zeta}, \boldsymbol{\nu}}^{(i)}$. We make the basic assumption there exists $i \in[K], \mathbf{w} \in \mathcal{W}_{K} \backslash\{\varnothing\}$ such that $\zeta^{(i)}(\mathbf{w})>0$ and $\nu_{i, \mathbf{w}}$ is not a Dirac mass, so that there is indeed some randomness in the spatial displacements, and say that the displacement laws $\nu$ are non-degenerate in this case.

\subsection{An invariance principle for SGW trees}

Let $\boldsymbol{\zeta}$ be an ordered offspring distribution, and $\boldsymbol{\mu}=p_{*} \boldsymbol{\zeta}$. For $1 \leq i, j, k \leq K$, define

$$
Q_{j k}^{(i)}=\sum_{\mathbf{z} \in \mathbb{Z}_{+}^{K}} z_{j} z_{k} \mu^{(i)}(\mathbf{z}), \quad j \neq k,
$$

and

$$
Q_{j j}^{(i)}=\sum_{\mathbf{z} \in \mathbb{Z}_{+}^{K}} z_{j}\left(z_{j}-1\right) \mu^{(i)}(\mathbf{z}) .
$$

We say that $\boldsymbol{\mu}$ (or $\boldsymbol{\zeta}$ ) has finite variance if $Q_{j k}^{(i)}<\infty$ for all $i, j, k \in[K]$. Under this assumption, for each $i$, $\left(Q_{j k}^{i}, 1 \leq j, k \leq K\right)$ is the matrix of a non-negative quadratic form on $\mathbb{R}^{K}$, which we call $Q^{(i)}(\mathbf{x}), \mathbf{x} \in \mathbb{R}^{K}$. Indeed, it can be interpreted as the Hessian matrix of the generating function of $\mu^{(i)}$ evaluated at $(1, \ldots, 1)$, as in Vatutin and Dyakonova (2001). We say that $\boldsymbol{\zeta}$, or $\boldsymbol{\mu}$, has small exponential moments if for some $\varepsilon>0$,

$$
\max _{i \in[K]} \boldsymbol{\zeta}^{(i)}(\exp (\varepsilon|\mathbf{w}|))=\max _{i \in[K]} \mu^{(i)}\left(\exp \left(\varepsilon|\mathbf{z}|_{1}\right)\right)<\infty
$$

This is obviously stronger than having finite variance.

Assuming $\mathfrak{M}_{\boldsymbol{\mu}}$ irreducible and $\boldsymbol{\mu}$ non-degenerate, critical and with finite variance, we let $\mathbf{a}, \mathbf{b}$ be the left and right eigenvectors of $M$ with eigenvalue 1 , chosen so that $|\mathbf{a}|_{1}=1$ and $\mathbf{a} \cdot \mathbf{b}=1$. Let

$$
\sigma=\sqrt{\sum_{i=1}^{K} a_{i} Q^{(i)}(\mathbf{b})}=\sqrt{\mathbf{a} \cdot \mathbf{Q}(\mathbf{b})}
$$

where $\mathbf{Q}(\mathbf{x})$ is the $K$-dimensional vector $\left(Q^{(i)}(\mathbf{x}), 1 \leq i \leq K\right)$. This should be interpreted as the 'variance' of the offspring distribution of the two-type process, as it plays a rôle similar to the variance for monotype GW processes in the asymptotics of the survival probability, see Vatutin and Dyakonova (2001).

For $\mathbf{t} \in \mathcal{T}$, we let $\varnothing=u(0) \prec u(1) \prec \ldots \prec u(\# \mathbf{t}-1)$ be the ordered list of vertices of $\mathbf{t}$, and let $\left(H_{n}^{\mathbf{t}}=|u(n)|, n \geq 0\right)$ be the height process of $\mathbf{t}$, with the convention that $|u(n)|=0$ for $n \geq \# \mathbf{t}$. For $\mathbf{t} \in \mathbb{T}^{(K)}$, let $\left(S_{n}^{\mathbf{t}}=\ell_{u(n)}, n \geq 0\right)$, with the convention that it equals 0 for $n \geq \# \mathbf{t}$.

Theorem 2 (Miermont (2006), Theorems 2 and 4) Let $\zeta$ be a critical non-degenerate ordered offspring distribution such that $\boldsymbol{\mu}=p_{*} \boldsymbol{\zeta}$ has small exponential moments. Let $\left(\nu_{i, \mathbf{w}}, i \in[K], \mathbf{w} \in \mathcal{W}_{K}\right)$ be nondegenerate spatial displacement distributions that are all centered, and admit moments $\mathrm{m}_{i, \mathbf{w}}=\nu_{i, \mathbf{w}}\left(|\mathbf{y}|_{2}^{8+\varepsilon}\right)$, for some $\varepsilon>0$ such that

$$
\sup _{i \in[K]} \mathrm{m}_{i, \mathbf{w}}=O\left(|\mathbf{w}|^{D}\right)
$$

for some $D>0$ (here, $|\mathbf{y}|_{2}$ is the Euclidean norm of $\left.\mathbf{y}\right)$. Write

$$
\Sigma=\sqrt{\sum_{i \in[K]} a_{i} \sum_{\mathbf{w} \in \mathcal{W}_{K}} \zeta^{(i)}(\mathbf{w}) \sum_{j=1}^{|\mathbf{w}|} b_{w_{j}} \nu_{i, \mathbf{w}}\left(y_{j}^{2}\right)}<\infty .
$$

Then for every $i, j \in[K]$, the following convergence in distribution holds on $\mathbb{D}([0,1], \mathbb{R})^{2}$ :

$$
\begin{gathered}
\left(\left(\frac{H_{[\# T t]}^{T}}{n^{1 / 2}}\right)_{0 \leq t \leq 1},\left(\frac{S_{[\# T t]}^{T}}{n^{1 / 4}}\right)_{0 \leq t \leq 1}\right) \text { under } \mathbb{P}^{(i)}\left(\cdot \mid \# T^{(j)}=n\right) \\
\underset{n \rightarrow \infty}{\stackrel{d}{\longrightarrow}}\left(\left(\frac{2}{\sigma \sqrt{a_{j}}} B_{t}^{\mathrm{ex}}\right)_{0 \leq t \leq 1},\left(\Sigma \sqrt{\frac{2}{\sigma \sqrt{a_{j}}}} R_{t}^{\mathrm{ex}}\right)_{0 \leq t \leq 1}\right)
\end{gathered}
$$


where $B^{\mathrm{ex}}, R^{\mathrm{ex}}$ is defined in Section 1.2

Moreover, if

$$
\Lambda_{i}^{\mathbf{t}}(k)=\#\{0 \leq j \leq k: e(u(j))=i\}, \quad 0 \leq k<\# \mathbf{t},
$$

and with the convention $\Lambda_{i}^{\mathbf{t}}(\# \mathbf{t})=\Lambda_{i}^{\mathbf{t}}(\# \mathbf{t}-1)$, then $\left(\Lambda_{i}^{T}([\# T t]) / \# T, 0 \leq t \leq 1\right)$ under $\mathbb{P}^{(i)}\left(\cdot \mid \# T^{(j)}=\right.$ $n)$ converges in probability to $\left(a_{j} t, 0 \leq t \leq 1\right)$ for the uniform norm.

Remark. The last statement shows that the relative frequencies of vertices of different kinds in large conditioned SGW trees are asymptotically deterministic. This gives a reason to believe that Theorem 2 remains true when conditioning on events like $\left\{\# T^{(i)}+T^{(j)}=n\right\}$ for some $i \neq j \in[K]$ instead of conditioning on only one type, though the proof might be technically involved. Recalling the third remark in Section 1.2, this would imply the statement of Theorem 1 to hold also when conditioning on the number of faces of the planar maps.

\subsection{Shuffling}

Let $\mathfrak{S}_{n}$ denote the set of bijections $[n] \rightarrow[n]$, and take some arbitrary convention for $\mathfrak{S}_{0}$. Let $\mathbf{t} \in \mathcal{T}^{(K)}$ be a $K$-type spatial tree, and suppose we are given a family $\boldsymbol{\pi}=\left(\pi_{u}, u \in \mathbf{t}\right)$ such that $\pi_{u} \in \mathfrak{S}_{c^{\mathbf{t}}(u)}, u \in \mathbf{t}$. We can define a tree $\boldsymbol{\pi}(\mathbf{t})$ that shuffles the children of $u$ with the help of $\pi_{u}$, by letting

$$
\boldsymbol{\pi}(\mathbf{t})=\left\{\boldsymbol{\pi}(u)=\pi_{\varnothing}\left(u_{1}\right) \pi_{u_{1}}\left(u_{2}\right) \ldots \pi_{u_{1} \ldots u_{n-1}}\left(u_{n}\right): u=u_{1} \ldots u_{n} \in \mathbf{t}\right\},
$$

which is naturally marked by the type function $\boldsymbol{\pi}(e)(\boldsymbol{\pi}(u))=e(u)$ and the spatial embedding $\boldsymbol{\pi}(\boldsymbol{\ell})=$ $\left(\boldsymbol{\pi}(\ell)_{v}=\ell_{\boldsymbol{\pi}^{-1}(v)}, v \in \boldsymbol{\pi}(\mathbf{t})\right)$ (the formalism is taken after Weill $(2005)$ ).

In general it holds that randomly shuffled $K$-type SGW trees remain SGW trees, as long as, informally, the children of different vertices $u \in \mathbf{t}$ are shuffled independently, in a way that depends only on $\mathbf{w}^{\mathbf{t}}(u)$. For our purposes, we will need the following particular version of this property. If $\mathbf{x}=\left(x_{1}, \ldots, x_{n}\right)$ is a finite sequence, we let $\overleftarrow{\mathbf{x}}=\left(x_{n-i+1}, 1 \leq i \leq n\right)$ be the reversed sequence. If $\lambda$ is a measure on $\mathbb{R}^{n}$, we naturally let $\overleftarrow{\lambda}$ be the push-forward of $\lambda$ by the mapping $\leftarrow: \mathbb{R}^{n} \rightarrow \mathbb{R}^{n}$. For $\mathbf{t} \in \mathcal{T}$ and $u \in \mathbf{t}$ with $c^{\mathbf{t}}(u) \geq 2$, take independent $\left(\Pi_{u}, u \in \mathbf{t}\right)$ such that $\Pi_{u}$ is either the identity element of $\mathfrak{S}_{c^{\mathbf{t}}(u)}$ or the involutive permutation $\left(c^{\mathbf{t}}(u)-i+1,1 \leq i \leq c_{u}^{\mathbf{t}}\right)$, with respective probabilities $1 / 2$ and $1 / 2$ (for convenience, we denote these two permutations by $I, J$ whatever $c^{\mathbf{t}}(u)$ is, even if it is 0$)$. Let $\Phi_{\mathbf{t}, e, \ell}(\cdot)$ be the law of the tree $\Pi(\mathbf{t}, e, \ell)$, so that each set of children of vertices is either kept as such, or taken in reversed order.

Lemma 1 Let $\boldsymbol{\mu}$ be an offspring distribution and $\boldsymbol{\zeta}$ its associated uniformly ordered offspring distribution, and let $\boldsymbol{\nu}$ be spatial displacement distributions. Then,

$$
\int_{\mathbb{T}^{(K)}} \mathbb{P}_{\boldsymbol{\zeta}, \boldsymbol{\nu}}^{(i)}(\mathrm{d}(\mathbf{t}, e, \boldsymbol{\ell})) \Phi_{\mathbf{t}, e, \ell}(\cdot)=\mathbb{P}_{\boldsymbol{\zeta}, \overleftrightarrow{\boldsymbol{\nu}}}^{(i)}(\cdot),
$$

where for $j \in[K],|\mathbf{w}| \in \mathcal{W}_{K}$,

$$
\overleftrightarrow{\nu}_{j, \mathbf{w}}(\mathrm{d} \mathbf{y})=\frac{\nu_{j, \mathbf{w}}(\mathrm{d} \mathbf{y})+\overleftarrow{\nu}_{j, \overleftarrow{\mathbf{w}}}(\mathrm{d} \mathbf{y})}{2}
$$

Proof. This is an easy consequence of the fact that, if $(\mathbf{t}, e) \in \mathcal{T}_{i}^{(K)}$ and $A_{v} \in \mathcal{B}\left(\mathbb{R}^{c^{\mathbf{t}}(v)}\right), v \in \mathbf{t}$, then the set $\left\{(\mathbf{t}, e, \ell):\left(y_{v i}, 1 \leq i \leq c^{\mathbf{t}}(v)\right)_{v \in \mathbf{t}} \in \prod_{v \in \mathbf{t}} A_{v}\right\}$ has a mass with respect to the measure of the left-hand side of 12 , which is given by

$$
\sum_{\left(\mathbf{t}^{\prime}, e^{\prime}, \ell^{\prime}\right) \in \mathbb{T}^{(K)}} \prod_{u \in \mathbf{t}^{\prime}} \zeta^{\left(e^{\prime}(u)\right)}\left(\mathbf{w}^{\mathbf{t}^{\prime}}(u)\right) \nu_{e^{\prime}(u), \mathbf{w}^{\mathbf{t}^{\prime}(u)}}\left(A_{u} \frac{\sum_{u \in \mathbf{t}^{\prime}, \pi_{u} \in\{I, J\}} \mathbb{1}_{\left\{\left(\mathbf{t}^{\prime}, e^{\prime}, \ell^{\prime}\right)=\boldsymbol{\pi}(\mathbf{t}, e, \ell)\right\}}}{2^{\# \mathbf{t}}}\right.
$$

where it should be understood that $\{I, J\}$ is a multiset with two elements, even in the case where $c^{\mathbf{t}}(u) \in$ $\{0,1\}$ (this is just for notational convenience). Since $\zeta$ is the uniform ordering of $\boldsymbol{\mu}, \zeta^{(i)}(\mathbf{w})$ depends only on $i$ and $p(\mathbf{w})$, so that $\zeta^{\left(e^{\prime}(u)\right)}\left(\mathbf{w}^{\mathbf{t}^{\prime}}(\boldsymbol{\pi}(u))\right)=\zeta^{(e(u))}\left(\mathbf{w}^{\mathbf{t}}(u)\right)$ whenever $\left(\mathbf{t}^{\prime}, e^{\prime}, \boldsymbol{\ell}^{\prime}\right)=\boldsymbol{\pi}(\mathbf{t}, e, \boldsymbol{\ell})$, and we can rewrite this as

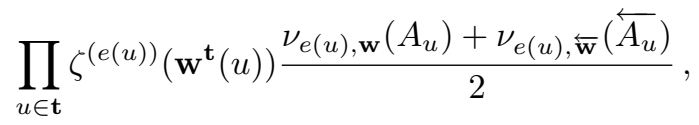

hence the result. 


\section{Relation between random maps and SGW trees}

We will now see how one can encode the $P_{\mathbf{q}}$-distributed maps in terms of SGW trees. Until the end of this section, we will only consider the case where $K=3$ with the notations of the previous section.

\subsection{The Bouttier-Di Francesco-Guitter bijection}

The Bouttier-Di Francesco-Guitter (BDFG) bijection (Bouttier et al. (2004)) yields a way to code elements of $\mathcal{M}_{+}$and $\mathcal{M}_{0}$ with the help of certain 3-type spatial trees, as we now explain. We consider the set $\overline{\mathcal{T}} \subset \mathcal{T}^{(3)}$ of 3-type trees, in which, for every $(\mathbf{t}, e) \in \overline{\mathcal{T}}$, and $u \in \mathbf{t}$,

1. if $e(u)=1$ then $\mathbf{z}^{\mathbf{t}}(u)=(0,0, k)$ for some $k \geq 0$ (so individuals of type 1 can only have children of type 3$)$,

2. if $e(u) \in\{2,3\}$, then $\mathbf{z}^{\mathbf{t}}(u)=\left(k, k^{\prime}, 0\right)$ for some $k, k^{\prime} \geq 0$ (so individuals of type 2 or 3 can only have children of types 1 and 2 ).

When drawing a picture, we represent individuals of type $1,2,3$ respectively by vertices with the three shapes $\bigcirc, \diamond, \bullet$. See Figure 4 for an example. Naturally, we let $\overline{\mathcal{T}}_{i}=\overline{\mathcal{T}} \cap \mathcal{T}_{i}^{(3)}$ for $i \in\{1,2,3\}$.

Next, we let $\overline{\mathbb{T}} \subset \mathbb{T}^{(3)}$ be the set of 3 -type spatial trees $(\mathbf{t}, e, \ell)$, with $(\mathbf{t}, e) \in \overline{\mathcal{T}}, \ell_{\varnothing}=0$, and such that the following labeling constraints hold: for every $u \in \mathbf{t}$,

3. $\ell_{u} \in \mathbb{Z}$,

4. if $e(u)=1$ then $\ell_{u i}=\ell_{u}$ for all $1 \leq i \leq c^{\mathbf{t}}(u)$, so that vertices of type 3 inherit the label of their fathers,

5. if $e(u) \in\{2,3\}$, and $c^{\mathbf{t}}(u)=k$, we let by convention $u 0=u=u \underline{k+1}$ (here $u \underline{k+1}$ means that $k+1$ is considered as a letter). Then, writing $x_{u i}=\ell_{u i}-\ell_{u i-1}, 1 \leq \overline{i \leq k}+1$, it holds that

(a) if $e(u \underline{i-1}) \in\{1,3\}$ then $x_{u i} \in \mathbb{Z}_{+} \cup\{-1\}$

(b) if $e(u \underline{i-1})=2$ then $x_{u i} \in \mathbb{Z}_{+}$.

Notice that $y_{u i}=\sum_{1 \leq j \leq i} x_{u j}$ for $1 \leq i \leq c^{\mathbf{t}}(u)$. We adopt the natural notation $\overline{\mathbb{T}}_{i}=\overline{\mathbb{T}} \cap \mathbb{T}_{i}^{(3)}$ for $i \in\{1,2,3\}$.

Proposition 2 (BDFG bijections) I. There exists a bijection $\Psi^{+}$from $\mathcal{M}_{+}$onto $\overline{\mathbb{T}}_{1}$, that sends $\dagger$ on $(\{\varnothing\}, e(\varnothing)=$ $\left.1, \ell_{\varnothing}=0\right)$ and satisfies the following extra properties. If $\mathbf{m} \in \mathcal{M}_{+} \backslash\{\dagger\}$ and $(\mathbf{t}, e, \ell)=\Psi^{+}(\mathbf{m})$,

(i) $\# F(\mathbf{m})=\# \mathbf{t}^{(2)}+\# \mathbf{t}^{(3)}$. Moreover, with every $u \in \mathbf{t}^{(2)}$ (resp. $\left.\mathbf{t}^{(3)}\right)$ such that $\mathbf{z}^{\mathbf{t}}(u)=\left(k, k^{\prime}, 0\right)$ is associated a unique face of $\mathbf{m}$ that has degree $2 k+k^{\prime}+1$ (resp. $\left.2 k+k^{\prime}+2\right)$.

(ii) Vertices $v \in V(\mathbf{m}) \backslash\{r\}$ at distance $d>0$ from the base vertex $r$ are in one-to-one correspondence with vertices $u \in \mathbf{t}^{(1)}$ with $\ell_{u}-\min _{\mathbf{t}^{(1)}} \ell+1=d$, where $\min _{\mathbf{t}^{(1)}} \ell:=\min _{u \in \mathbf{t}^{(1)}} \ell_{u}$ ( similar notations are adopted in the sequel). In particular, $\# V(\mathbf{m})=\# \mathbf{t}^{(1)}+1$,

$$
\mathcal{R}(\mathbf{m})=\max _{\mathbf{t}^{(1)}} \boldsymbol{\ell}-\min _{\mathbf{t}^{(1)}} \boldsymbol{\ell}+1
$$

and

$$
\mathcal{J}^{\mathbf{m}}(k)=\frac{1}{\# \mathbf{t}^{(1)}+1}\left(\#\left\{u \in \mathbf{t}^{(1)}: \ell_{u}-\min _{\mathbf{t}^{(1)}} \boldsymbol{\ell}+1=k\right\}+\mathbb{1}_{\{k=0\}}\right), \quad k \geq 0 .
$$

II. There exists a bijection $\Psi^{0}$ from $\mathcal{M}_{0}$ onto the set $\overline{\mathbb{T}}_{2} \times \overline{\mathbb{T}}_{2}$. If $\mathbf{m} \in \mathcal{M}_{0}$ and $\Psi^{0}(\mathbf{m})=\left((\mathbf{t}, \ell),\left(\mathbf{s}, \ell^{\prime}\right)\right)$, then similarly as above,

(i)' faces of $\mathbf{m}$ are in one-to-one correspondence with vertices of type 2 and 3 of the (disjoint) union of $\mathbf{t}$ and $\mathbf{s}$, so that $\# F(\mathbf{m})=\# \mathbf{t}^{(2)}+\# \mathbf{t}^{(3)}+\# \mathbf{s}^{(2)}+\# \mathbf{s}^{(3)}$, in such a way that with every $u \in \mathbf{t}^{(2)}$ (resp. $\left.\mathbf{t}^{(3)}\right)$ such that $\mathbf{z}^{\mathbf{t}}(u)=\left(k, k^{\prime}, 0\right)$ is associated a unique face of $\mathbf{m}$ that has degree $2 k+k^{\prime}+1$ (resp. $2 k+k^{\prime}+2$ ), and similarly for $u \in \mathbf{s}$. 
(ii)' Vertices $v \in V(\mathbf{m}) \backslash\{r\}$ at distance $d>0$ from the base vertex, if any, are in one-to-one correspondence with elements $u$ of the disjoint union of $\mathbf{t}^{(1)}$ and $\mathbf{s}^{(1)}$, with $\ell_{u}-\min _{\mathbf{t}^{(1)}} \boldsymbol{\ell} \wedge \min _{\mathbf{s}^{(1)}} \ell^{\prime}+1=d$ or $\ell_{u}^{\prime}-\min _{\mathbf{t}^{(1)}} \ell \wedge \min _{\mathbf{s}^{(1)}} \ell^{\prime}+1=d$ accordingly. In particular, $\# V(\mathbf{m})=\# \mathbf{t}^{(1)}+\# \mathbf{s}^{(1)}+1$,

$$
\mathcal{R}(\mathbf{m})=\max \left(\max _{\mathbf{t}^{(1)}} \boldsymbol{\ell}-\min _{\mathbf{t}^{(1)}} \boldsymbol{\ell} \wedge \min _{\mathbf{s}^{(1)}} \boldsymbol{\ell}^{\prime}+1, \max _{\mathbf{s}^{(1)}} \boldsymbol{\ell}^{\prime}-\min _{\mathbf{t}^{(1)}} \boldsymbol{\ell} \wedge \min _{\mathbf{s}^{(1)}} \boldsymbol{\ell}_{u}^{\prime}+1\right)
$$

and

$$
\begin{aligned}
\mathcal{J}^{\mathbf{m}}(k)= & \frac{1}{\# \mathbf{t}^{(1)}+\# \mathbf{s}^{(1)}+1}\left(\#\left\{u \in \mathbf{t}^{(1)}: \ell_{u}-\min _{\mathbf{t}^{(1)}} \boldsymbol{\ell} \wedge \min _{\mathbf{s}^{(1)}} \boldsymbol{\ell}^{\prime}+1=k\right\}\right. \\
& \left.+\#\left\{u \in \mathbf{s}^{(1)}: \ell_{u}^{\prime}-\min _{\mathbf{t}^{(1)}} \boldsymbol{\ell} \wedge \min _{\mathbf{s}^{(1)}} \boldsymbol{\ell}^{\prime}+1=k\right\}+\mathbb{1}_{\{k=0\}}\right), \quad k \geq 0 .
\end{aligned}
$$

Proof. This statement might look quite different from that of (Bouttier et al., 2004, Sect. 4.2), but is in fact equivalent. Indeed, the original bijection from Bouttier et al. (2004) holds between maps that are solely pointed, and not rooted, and with decorated unrooted planar trees (called mobiles) with three types of vertices: labelled vertices $\bigcirc$ and $\diamond$, and unlabelled vertices $\circ$, such that $\circ$ vertices are adjacent to both other kinds of vertices, while $\diamond$ vertices have exactly two neighbors, both of type $\circ$. The labels are all integers greater than or equal to 1 , and have to check the following constraint. Let $v_{1}, \ldots, v_{k}$ be the vertices that are displayed around a vertex of type $\circ$, arranged in anti-trigonometric order, and let $l_{1}, \ldots, l_{k}$ be their labels. Then the label differences $l_{i+1} \bmod k-l_{i}, 1 \leq i \leq k$ obviously have sum 0 , and belong to $\mathbb{Z}_{+} \cup\{-1\}$ if $v_{i}$ is of type $\bigcirc$, and to $\mathbb{Z}_{+}$if $v_{i}$ is of type $\diamond$. This constraint is illustrated in Figure 2

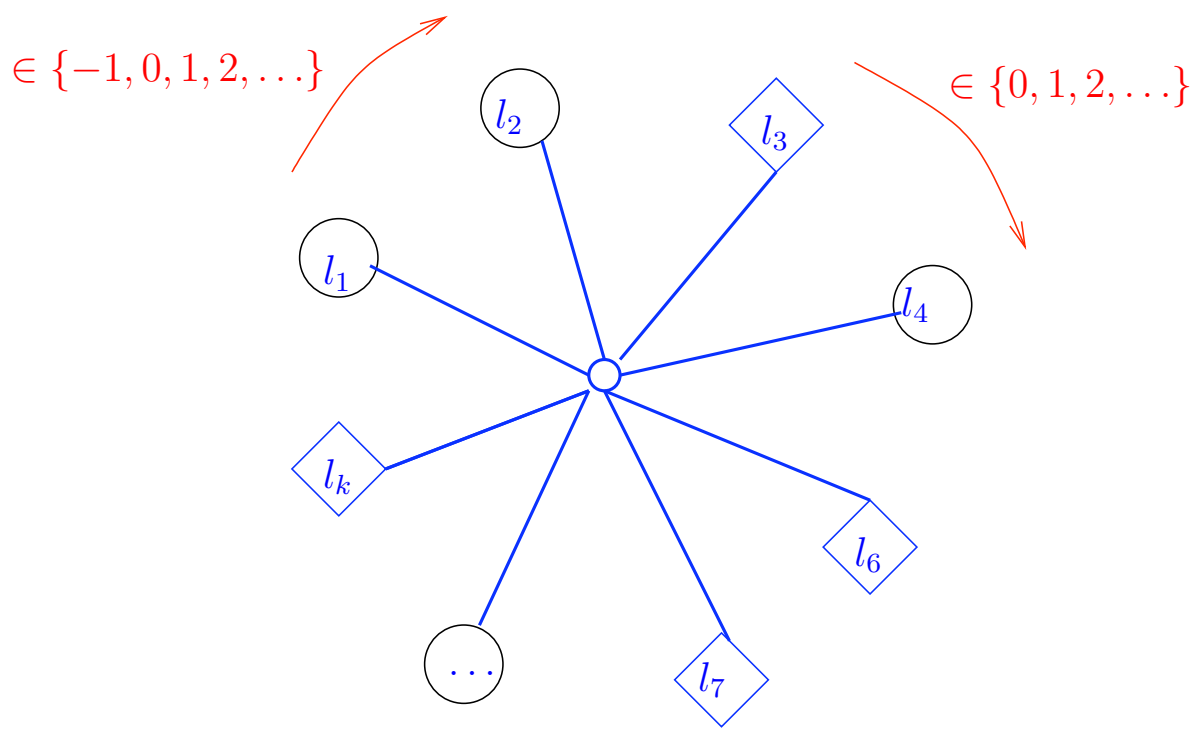

Fig. 2: Labeling constraint around o vertices of mobiles. In this configuration, we obtain $l_{2}$ from $l_{1}$ (resp. $l_{4}$ from $l_{3}$ ) by adding an integer in $\mathbb{Z}_{+} \cup\{1\}$ (resp. $\mathbb{Z}_{+}$)

It is explained at the end of (Bouttier et al. 2004, Sect. 4.2) how a further rooting of the map allows to turn the 'mobile' into a rooted object. If the root $\vec{e}$ satisfies $d_{\mathbf{m}}\left(e_{-}, r\right)+1=d_{\mathbf{m}}\left(e_{+}, r\right)$, i.e. if the map is an element of $\mathcal{M}_{+}$, then the mobile can be rooted at a vertex of type $\bigcirc$. Moreover, one lifts the positivity constraint on labels by subtracting the label of the root to all other labels. This gives in particular the property (ii) of correspondence between $\bigcirc$ vertices and $V(\mathbf{m})$. To obtain our exact formulation, it suffices to do the following modification to the rooted tree thus obtained.

First, for every type-o vertex with father of type $\diamond$, we modify the tree by erasing the $\circ$ vertex and connecting its children directly to its $\diamond$-type father, in the same planar order. Thus, the only o vertices that remain are the children of $\bigcirc$ vertices.

Next, all remaining o vertices earn the label of their $\bigcirc$-type father.

Last, we do a cosmetic arrangement by changing $\circ$ vertices into $\bullet$ s. The labeled trees thus obtained are exactly those of $\overline{\mathbb{T}}_{1}$, as the reader will check (note that the label constraints explained above precisely amount to properties 5. (a) and (b) of the definition of $\overline{\mathbb{T}}$ ). Moreover, in the original mobile, o vertices with 


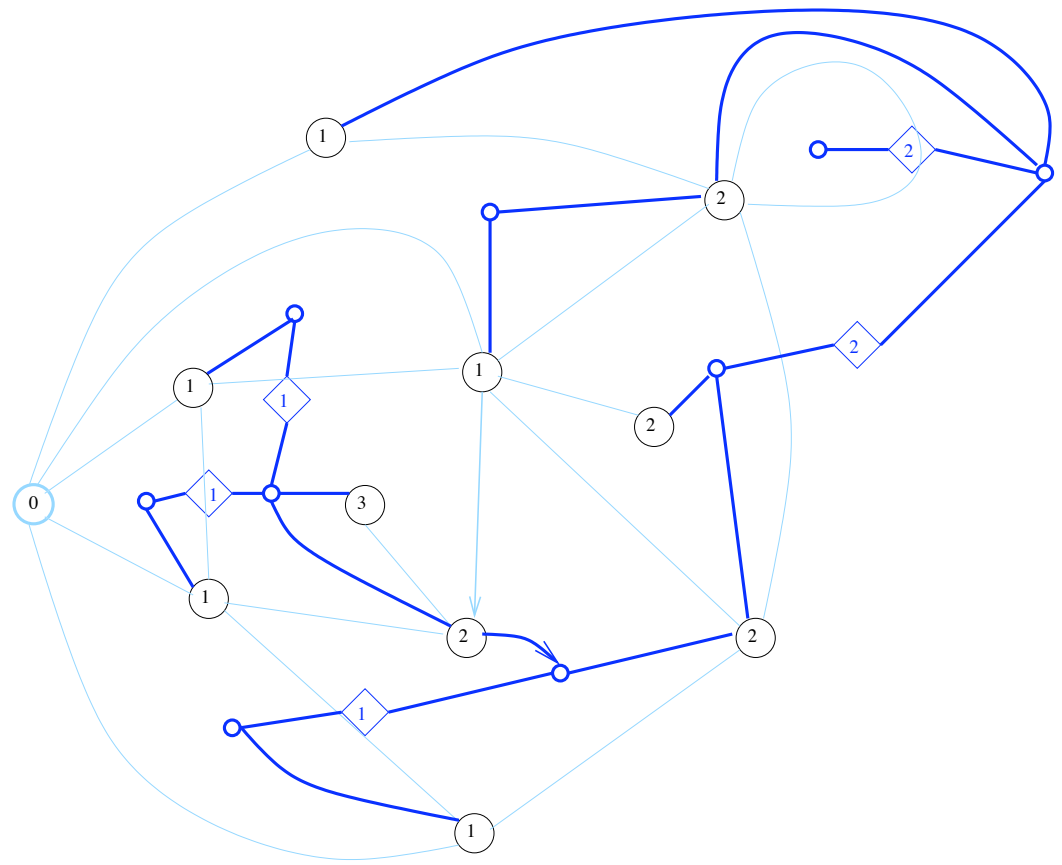

Fig. 3: The 'mobile' associated with the map of Figure 1 which is represented with pale lines. The arrow points out from the root of the tree towards its first child. It still remains to lift the positivity constraint on labels by subtracting the label of the root, namely 2 , to all the others.

$k$ neighbours of type $\bigcirc$ and $k^{\prime}$ neighbours of type $\diamond$ correspond in a one-to-one way to faces of $\mathbf{m}$ with degree $2 k+k^{\prime}$. This amounts to property (i) of the statement.

The statement for $\mathcal{M}_{0}$ is obtained in a similar way, noticing that when the root of the map connects two vertices at equal distance from the base vertex, then one has to consider a pair of 'half-mobiles' which are both naturally rooted at a vertex of type ' $\diamond$ '. One lifts the positivity constraint by subtracting the label of this vertex to all other labels, and do the same cosmetic changes as above.

\subsection{Description of the push-forward measures $\Psi_{*}^{+} P_{\mathbf{q}}^{+}$and $\Psi_{*}^{0} P_{\mathbf{q}}^{0}$}

The key observation states that the image of Boltzmann-distributed maps under the BDFG bijections are essentially SGW trees. In order to prove this, we first need to count the number of possible labelings $\ell=\left(\ell_{u}, u \in \mathbf{t}\right)$ of a given tree $(\mathbf{t}, e) \in \overline{\mathcal{T}}$ which are such that $(\mathbf{t}, e, \ell) \in \overline{\mathbb{T}}$. To this end, since we know that $\ell_{\varnothing}=0$, it suffices to find the number of possible label differences $\left(y_{u i}=\ell_{u i}-\ell_{u}, 1 \leq i \leq c^{\mathrm{t}}(u)\right)$ for all $u \in \mathbf{t}$. Equivalently, it suffices to find the number of possible sequences $\left(x_{u i}=\ell_{u i}-\ell_{u i-1}, 1 \leq\right.$ $\left.i \leq c^{\mathbf{t}}(u)+1\right)$, with our usual convention $u 0=u=u c^{\mathbf{t}}(u)+1$. If $u \in \mathbf{t}^{(1)}$, we know that its children, all of type 3 , earn the label $\ell_{u}$, so there is only one possibility. If $u \in \mathbf{t}^{(3)}$ has children $\mathbf{w}^{\mathbf{t}}(u)=\mathbf{w} \in \mathcal{W}_{3}$, with say $\left(z_{1}^{\mathbf{t}}(u), z_{2}^{\mathbf{t}}(u)\right)=\left(k, k^{\prime}\right)$, then we know from property 5 . (a) in the definition of $\overline{\mathbb{T}}$ that the possible sequences $\left(x_{u i}+\mathbb{1}_{\left\{w_{i-1}^{\mathbf{t}}(u)=1\right\}}, 1 \leq i \leq c^{\mathbf{t}}(u)+1\right)$, with the convention $w_{0}^{\mathbf{t}}(u)=1$, are the elements of the set

$$
\left\{\left(n_{1}, \ldots, n_{k+k^{\prime}+1}\right) \in \mathbb{Z}_{+}^{k+k^{\prime}+1}: n_{1}+\ldots+n_{k+k^{\prime}+1}=k+1\right\} .
$$

Notice that by adding 1 to every term of a sequence in the set 17 , we obtain a sequence of $k+k^{\prime}+1$ positive integers with sum $2 k+k^{\prime}+2$. It is a classical exercise to check that there are exactly $\left(\begin{array}{c}2 k+k^{\prime}+1 \\ k+1\end{array}\right)=N_{\bullet}\left(k, k^{\prime}\right)$ such sequences (recall (1)).

Similarly, if $u \in \mathbf{t}^{(2)}$ has children $\mathbf{w}^{\mathbf{t}}(u)=\mathbf{w} \in \mathcal{W}_{3}$, with $\left(z_{1}^{\mathbf{t}}(u), z_{2}^{\mathbf{t}}(u)\right)=\left(k, k^{\prime}\right)$, then the possible sequences $\left(x_{u i}+\mathbb{1}_{\left\{w_{i-1}^{\mathrm{t}}(u)=1\right\}}, 1 \leq i \leq c^{\mathrm{t}}(u)+1\right)$, where this time $w_{0}^{\mathrm{t}}(u)=2$, are the elements of the set

$$
\left\{\left(n_{1}, \ldots, n_{k+k^{\prime}+1}\right) \in \mathbb{Z}_{+}^{k+k^{\prime}+1}: n_{1}+\ldots+n_{k+k^{\prime}+1}=k\right\},
$$

which has $N_{\diamond}\left(k, k^{\prime}\right)$ elements. The number of possible labellings $\ell$ so that $(\mathbf{t}, e, \ell) \in \mathbb{T}$ for a given $(\mathbf{t}, e) \in \overline{\mathcal{T}}$ is thus

$$
\prod_{u \in \mathbf{t}^{(2)}} N_{\diamond}\left(z_{1}^{\mathbf{t}}(u), z_{2}^{\mathbf{t}}(u)\right) \prod_{u \in \mathbf{t}^{(3)}} N_{\bullet}\left(z_{1}^{\mathbf{t}}(u), z_{2}^{\mathbf{t}}(u)\right) .
$$




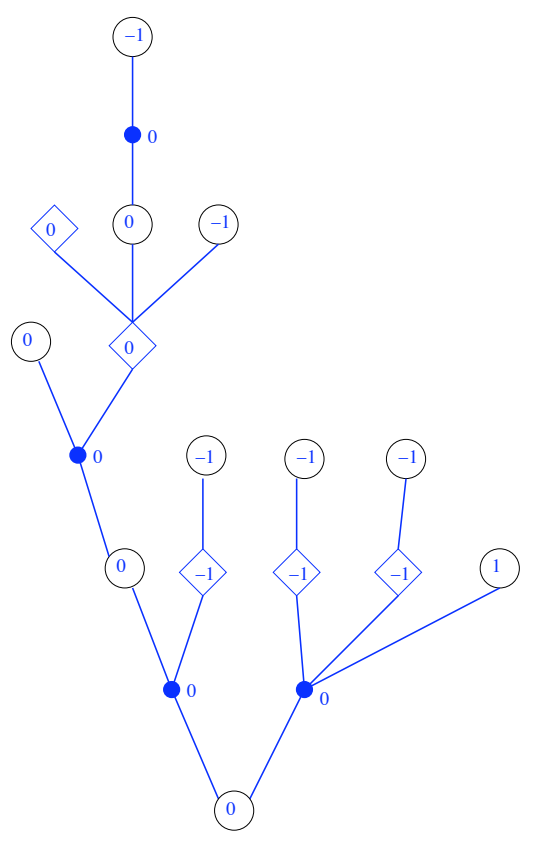

Fig. 4: The spatial 3-type tree obtained by the BDFG bijection from the mobile of Figure 3 after the cosmetic changes of the proof of Proposition 2 The above spatial tree is a typical element of $\overline{\mathbb{T}}_{1}$.

Finally, for every $\mathbf{w} \in \mathcal{W}_{3}$, let $\nu_{1, \mathbf{w}}^{0}$ be the Dirac mass at $0 \in \mathbb{R}^{|\mathbf{w}|}$. If $p(\mathbf{w})=\left(k, k^{\prime}, 0\right)$, we let $\nu_{2, \mathbf{w}}^{0}$ be the law on $\mathbb{Z}^{|\mathbf{w}|}$ of $\left(\sum_{i=1}^{j} X_{j}, 1 \leq j \leq k+k^{\prime}\right)$, where $\left(X_{j}+\mathbb{1}_{\left\{w_{j-1}=1\right\}}, 1 \leq j \leq k+k^{\prime}+1\right)$ is uniform on the set 18 with the convention $w_{0}=2$. We also let $\nu_{3, \mathrm{w}}^{0}$ be the law of $\left(\sum_{i=1}^{j} X_{i}, 1 \leq j \leq k+k^{\prime}\right)$, where $\left(X_{j}+\mathbb{1}_{\left\{w_{j-1}=1\right\}}, 1 \leq j \leq k+k^{\prime}+1\right)$ is uniform on the set $(17)$, where this time $w_{0}=1$. We take an arbitrary convention for $\nu_{i, \mathbf{w}}^{0}$ for $i \in\{2,3\}$ and $\mathbf{w}$ such that $p_{3}(\mathbf{w}) \neq 0$. As easily follows from the above discussion, $\nu_{i, \mathbf{w}}^{0}$ is the uniform law on the set of label differences $\left(\ell_{u j}-\ell_{u}, 1 \leq j \leq c^{\mathrm{t}}(u)\right)$ that can be attributed to a vertex $u \in \mathbf{t}$ with $e(u)=i$ and $\mathbf{w}^{\mathbf{t}}(u)=\mathbf{w}$ (where $(\mathbf{t}, e) \in \overline{\mathcal{T}}$ ), so that $(\mathbf{t}, e, \ell) \in \overline{\mathbb{T}}$.

Proposition 3 Let $\mathbf{q}$ be an admissible weight sequence and recall that by definition $Z_{\mathbf{q}}^{+}=W_{\mathbf{q}}\left(\mathcal{M}_{+}\right)$. Then there exists a constant $Z_{\mathbf{q}}^{\diamond}$ such that $\left(Z_{\mathbf{q}}^{+}, Z_{\mathbf{q}}^{\diamond}\right)$ is the 'good' solution to $(4)$. Define three probability distributions on $\mathbb{Z}_{+}^{3}$ by

$$
\mu^{(1)}(0,0, k)=\frac{1}{Z_{\mathbf{q}}^{+}}\left(1-\frac{1}{Z_{\mathbf{q}}^{+}}\right)^{k}, \quad k \geq 0,
$$

so under $\mu^{(1)}, \mathbf{z} \mapsto z_{3}$ has geometric law with parameter $1-\left(Z_{\mathbf{q}}^{+}\right)^{-1}$,

$$
\begin{aligned}
& \mu^{(2)}\left(k, k^{\prime}, 0\right)=\frac{\left(Z_{\mathbf{q}}^{+}\right)^{k}\left(Z_{\mathbf{q}}^{\diamond}\right)^{k^{\prime}} N_{\diamond}\left(k, k^{\prime}\right)\left(\begin{array}{c}
k+k^{\prime} \\
k
\end{array}\right) q_{1+2 k+k^{\prime}}}{f_{\mathbf{q}}^{\diamond}\left(Z_{\mathbf{q}}^{+}, Z_{\mathbf{q}}^{\diamond}\right)}, \quad k, k^{\prime} \geq 0, \\
& \mu^{(3)}\left(k, k^{\prime}, 0\right)=\frac{\left(Z_{\mathbf{q}}^{+}\right)^{k}\left(Z_{\mathbf{q}}^{\diamond}\right)^{k^{\prime}} N_{\bullet}\left(k, k^{\prime}\right)\left(\begin{array}{c}
k+k^{\prime} \\
k
\end{array}\right) q_{2+2 k+k^{\prime}}}{f_{\mathbf{q}}^{\bullet}\left(Z_{\mathbf{q}}^{+}, Z_{\mathbf{q}}^{\diamond}\right)}, \quad k, k^{\prime} \geq 0,
\end{aligned}
$$

and let $\zeta=\left(\zeta^{(1)}, \zeta^{(2)}, \zeta^{(3)}\right)$ be their uniformly ordered versions on $\mathcal{W}_{3}$. Then, the 3-type $G W$ tree $P_{\mu}^{(1)}$ is (sub)-critical, $\Psi_{*}^{+} P_{\mathbf{q}}^{+}=\mathbb{P}_{\boldsymbol{\zeta}, \boldsymbol{\nu}^{0}}^{(1)}$, where $\boldsymbol{\nu}^{0}=\left(\nu_{i, \mathbf{w}}^{0}, i \in[3], \mathbf{w} \in \mathcal{W}_{3}\right)$ was defined above.

Moreover, the weight sequence $\mathbf{q}$ is critical if and only if $\boldsymbol{\mu}$ is, and regular critical if and only if $\boldsymbol{\mu}$ further has small exponential moments.

Last, the probability measure $\Psi_{*}^{0} P_{\mathbf{q}}^{0}$ can be described as follows: the image of a $P_{\mathbf{q}}^{0}$-distributed random map under $\Psi^{0}$ is a pair of independent $S G W$ trees with same distribution $\mathbb{P}_{\boldsymbol{\zeta}, \nu^{0}}^{(2)}$.

Proof. Let $\mathbf{q}$ be a weight sequence, and recall the definition of $W_{\mathbf{q}}$ in Section 1.1 By (i) in Proposition 2 , if $\mathbf{m} \in \mathcal{M}_{+},(\mathbf{t}, e, \ell)=\Psi^{+}(\mathbf{m}) \in \overline{\mathbb{T}}_{1}$,

$$
W_{\mathbf{q}}\left(\left\{\left(\Psi^{+}\right)^{-1}(\mathbf{t}, e, \ell)\right\}\right)=\prod_{u \in \mathbf{t}^{(2)}} q_{1+2 z_{1}^{\mathbf{t}}(u)+z_{2}^{\mathbf{t}}(u)} \prod_{u \in \mathbf{t}^{(3)}} q_{2+2 z_{1}^{\mathbf{t}}(u)+z_{2}^{\mathbf{t}}(u)} .
$$


By summing over possible labellings $\ell$ with $(\mathbf{t}, e)$ fixed, and by 19, , we see that

$$
\begin{aligned}
& W_{\mathbf{q}}^{(1)}(\{\mathbf{t}, e\}):=W_{\mathbf{q}}\left(\left(\Psi^{+}\right)^{-1}\left\{\left(\mathbf{t}^{\prime}, e^{\prime}, \ell\right) \in \overline{\mathbb{T}}_{1}:\left(\mathbf{t}^{\prime}, e^{\prime}\right)=(\mathbf{t}, e)\right\}\right) \\
& \quad=\prod_{u \in \mathbf{t}^{(2)}} q_{1+2 z_{1}^{\mathbf{t}}(u)+z_{2}^{\mathbf{t}}(u)} N_{\diamond}\left(z_{1}^{\mathbf{t}}(u), z_{2}^{\mathbf{t}}(u)\right) \prod_{u \in \mathbf{t}^{(3)}} q_{2+2 z_{1}^{\mathbf{t}}(u)+z_{2}^{\mathbf{t}}(u)} N_{\bullet}\left(z_{1}^{\mathbf{t}}(u), z_{2}^{\mathbf{t}}(u)\right),
\end{aligned}
$$

which defines a measure $W_{\mathbf{q}}^{(1)}$ on $\overline{\mathcal{T}}_{1}$, with total mass $Z_{\mathbf{q}}^{+}$. We define measures $W_{\mathbf{q}}^{(2)}, W_{\mathbf{q}}^{(3)}$ on $\overline{\mathcal{T}}_{2}, \overline{\mathcal{T}}_{3}$ by mimicking formula $\sqrt{21}$, and let $Z_{\mathbf{q}}^{\diamond}$ resp. $Z_{\mathbf{q}}^{\bullet}$ be their respective total masses. Since an element of $\overline{\mathcal{T}}_{1}$ is rooted at a type- 1 vertex, which has a non-negative number of children all of type 3 , we obtain that

$$
Z_{\mathbf{q}}^{+}=\sum_{k \geq 0} \sum_{\mathbf{t}^{1}, \ldots, \mathbf{t}^{k} \in \overline{\mathcal{T}}_{3}} W_{\mathbf{q}}^{(3)}\left(\left\{\mathbf{t}^{1}\right\}\right) \times \ldots \times W_{\mathbf{q}}^{(3)}\left(\left\{\mathbf{t}^{k}\right\}\right)=\sum_{k \geq 0}\left(Z_{\mathbf{q}}^{\bullet}\right)^{k},
$$

so that $0<Z_{\mathbf{q}}^{\bullet}<1$ and $Z_{\mathbf{q}}^{+}=1 /\left(1-Z_{\mathbf{q}}^{\bullet}\right)$ whenever $Z_{\mathbf{q}}^{+}<\infty$.

Now, when an individual of type 3 gives birth to $k$ individuals of type 1 and $k^{\prime}$ individuals of type 2 , it contributes to $W_{\mathbf{q}}^{(3)}$ by a factor $q_{2+2 k+k^{\prime}} N_{\bullet}\left(k, k^{\prime}\right)$, and we obtain that

$$
\begin{aligned}
Z_{\mathbf{q}}^{\bullet} & =\sum_{k, k^{\prime} \geq 0} q_{2+2 k+k^{\prime}} N_{\bullet}\left(k, k^{\prime}\right) \sum_{\mathbf{w} \in \mathcal{W}_{3}: p(\mathbf{w})=\left(k, k^{\prime}, 0\right)}\left(Z_{\mathbf{q}}^{+}\right)^{k}\left(Z_{\mathbf{q}}^{\diamond}\right)^{k^{\prime}} \\
& =\sum_{k, k^{\prime} \geq 0} q_{2+2 k+k^{\prime}} N_{\bullet}\left(k, k^{\prime}\right)\left(\begin{array}{c}
k+k^{\prime} \\
k
\end{array}\right)\left(Z_{\mathbf{q}}^{+}\right)^{k}\left(Z_{\mathbf{q}}^{\diamond}\right)^{k^{\prime}}=f_{\mathbf{q}}^{\bullet}\left(Z_{\mathbf{q}}^{+}, Z_{\mathbf{q}}^{\diamond}\right),
\end{aligned}
$$

so that if $\mathbf{q}$ is admissible, $Z_{\mathbf{q}}^{\diamond}$ must be finite and satisfy $1-\left(Z_{\mathbf{q}}^{+}\right)^{-1}=f_{\mathbf{q}}^{\bullet}\left(Z_{\mathbf{q}}^{+}, Z_{\mathbf{q}}^{\diamond}\right)$. Here we have used the fact that $q_{k}>0$ for some $k>1$. Finally, for the same reasons, it holds that $Z_{\mathbf{q}}^{\diamond}=f_{\mathbf{q}}^{\diamond}\left(Z_{\mathbf{q}}^{+}, Z_{\mathbf{q}}^{\diamond}\right)$, so $Z_{\mathbf{q}}^{\diamond}$ is non-zero by the hypothesis that $q_{k}>0$ for some odd $k \geq 3$, and we see that $\left(Z_{\mathbf{q}}^{+}, Z_{\mathbf{q}}^{\diamond}\right)$ satisfies $(4)$. Next, we claim that for $\mathbf{t} \in \overline{\mathcal{T}}_{1}$,

$$
\prod_{u \in \mathbf{t}^{(1)}} \frac{\left(Z_{\mathbf{q}}^{\bullet}\right)^{z_{3}^{\mathbf{t}}(u)}}{Z_{\mathbf{q}}^{+}} \prod_{u \in \mathbf{t}^{(2)}} \frac{\left(Z_{\mathbf{q}}^{+}\right)^{z_{1}^{\mathbf{t}}(u)}\left(Z_{\mathbf{q}}^{\diamond}\right)^{z_{2}^{\mathbf{t}}(u)}}{f_{\mathbf{q}}^{\diamond}\left(Z_{\mathbf{q}}^{+}, Z_{\mathbf{q}}^{\diamond}\right)} \prod_{u \in \mathbf{t}^{(3)}} \frac{\left(Z_{\mathbf{q}}^{+}\right)^{z_{1}^{\mathbf{t}}(u)}\left(Z_{\mathbf{q}}^{\diamond}\right)^{z_{2}^{\mathbf{t}}(u)}}{f_{\mathbf{q}}^{\bullet}\left(Z_{\mathbf{q}}^{+}, Z_{\mathbf{q}}^{\diamond}\right)}=\frac{1}{Z_{\mathbf{q}}^{+}} .
$$

Indeed, notice that by definition of $\overline{\mathcal{T}}_{1}$, it holds that $\# \mathbf{t}^{(1)}-1=\sum_{u \in \mathbf{t}^{(2)} \cup \mathbf{t}^{(3)}} z_{1}^{\mathbf{t}}(u)$, while $\# \mathbf{t}^{(2)}=$ $\sum_{u \in \mathbf{t}^{(2)} \cup \mathbf{t}^{(3)}} z_{2}^{\mathbf{t}}(u)$ and $\# \mathbf{t}^{(3)}=\sum_{u \in \mathbf{t}^{(1)}} z_{3}^{\mathbf{t}}$ (in the first sum, we count all vertices of type 1 but the root, which is the child of no vertex). Using $f_{\mathbf{q}}^{\bullet}\left(Z_{\mathbf{q}}^{+}, Z_{\mathbf{q}}^{\diamond}\right)=Z_{\mathbf{q}}^{\bullet}$ and $Z_{\mathbf{q}}^{\diamond}=f_{\mathbf{q}}^{\diamond}\left(Z_{\mathbf{q}}^{+}, Z_{\mathbf{q}}^{\diamond}\right)$ yields the desired simplifications. Using this and the definition of $\boldsymbol{\mu}$ in the statement, we may re-express (21) as

$$
\frac{W_{\mathbf{q}}^{(1)}(\{\mathbf{t}, e\})}{Z_{\mathbf{q}}^{+}}=\prod_{u \in \mathbf{t}} \frac{\mu^{(e(u))}\left(\mathbf{z}^{\mathbf{t}}(u)\right)}{\# p^{-1}\left(\mathbf{z}^{\mathbf{t}}(u)\right)}=\prod_{u \in \mathbf{t}} \zeta^{(e(u))}\left(\mathbf{w}^{\mathbf{t}}(u)\right)=P_{\zeta}^{(1)}(\{\mathbf{t}, e\}),
$$

where the factor $\# p^{-1}\left(\mathbf{z}^{\mathbf{t}}(u)\right)$ is 1 whenever $e(u)=1$, and $\left(\begin{array}{c}z_{1}^{\mathbf{t}}(u)+z_{2}^{\mathbf{t}}(u) \\ z_{1}^{\mathbf{t}}(u)\end{array}\right)$ whenever $e(u) \in\{2,3\}$. Finally, redisplaying 20 in a similar way, we see that for every $(\mathbf{t}, e, \ell) \in \overline{\mathbb{T}}_{1}$,

$$
P_{\mathbf{q}}\left(\left\{\left(\Psi^{+}\right)^{-1}(\mathbf{t}, e, \ell)\right\}\right)=\prod_{u \in \mathbf{t}} \zeta^{(e(u))}\left(\mathbf{w}^{\mathbf{t}}(u)\right) \nu_{e(u), \mathbf{w}^{\mathbf{t}}(u)}^{0}\left(\left\{y_{u i}, 1 \leq i \leq c^{\mathbf{t}}(u)\right\}\right)
$$

giving the claim that $\Psi_{*}^{+} P_{\mathbf{q}}^{+}=\mathbb{P}_{\zeta, \nu^{0}}^{(1)}$.

To end the proof, notice that the matrix $\mathfrak{M}_{\mathbf{q}}$ of 6 is exactly the mean matrix $\mathfrak{M}_{\boldsymbol{\mu}}$ for $\boldsymbol{\mu}$. Since the trees $\Psi^{+}(\mathbf{m})$ are all finite by definition, it must hold that $\boldsymbol{\mu}$ is (sub)-critical (and non-degenerate), whence $\left(Z_{\mathbf{q}}^{+}, Z_{\mathbf{q}}^{\diamond}\right)$ has to be a 'good' solution of 4 . Obtaining that $\mathbf{q}$ is critical if and only if $\boldsymbol{\mu}$ is, is now just a matter of definitions, and the statement on regular criticality is straightforward from the definitions.

The study of $\Psi_{*}^{0} P_{\mathbf{q}}^{0}$ is analogous, keeping in mind that the bijection $\Psi^{0}$ associates a pair of trees (rooted at a ' $\diamond$ ' vertex) with one planar map. When computing the weights as above, we thus obtain that $Z_{\mathbf{q}}^{0}=\left(Z_{\mathbf{q}}^{\diamond}\right)^{2}$.

Proof of Proposition 1 In view of the previous proof, it remains to prove that if (4) has a good solution, then it is unique and $\mathbf{q}$ is admissible. Hence, assume that $\left(z^{+}, z^{\diamond}\right)$ is such a solution. Define probability measures $\overline{\boldsymbol{\mu}}, \overline{\boldsymbol{\zeta}}$ as in the statement of Proposition 3 , only replacing $\left(Z_{\mathbf{q}}^{+}, Z_{\mathbf{q}}^{\diamond}\right)$ by $\left(z^{+}, z^{\diamond}\right)$ everywhere. As 
in the previous proof, the mean matrix of $\bar{\mu}$ is exactly $\mathfrak{M}_{\mathbf{q}}\left(z^{+}, z^{\diamond}\right)$ defined at $\left(6\right.$. Since $\left(z^{+}, z^{\diamond}\right)$ is a good solution, the measure $\overline{\boldsymbol{\mu}}$ is (sub)-critical, and using a variant of $(22)$, also replacing $\left(Z_{\mathbf{q}}^{+}, Z_{\mathbf{q}}^{\diamond}\right)$ by $\left(z^{+}, z^{\diamond}\right)$, we see that the formula

$$
\frac{W_{\mathbf{q}}^{(1)}(\{\mathbf{t}, e\})}{z^{+}}=\prod_{u \in \mathbf{t}} \bar{\zeta}^{(e(u))}\left(\mathbf{w}^{\mathbf{t}}(u)\right)=P_{\overline{\boldsymbol{\zeta}}}^{(1)}(\{\mathbf{t}, e\}), \quad \mathbf{t} \in \overline{\mathcal{T}}_{1},
$$

holds (recall the notations $W_{\mathbf{q}}^{(i)}$ of the previous proof). From this, we get that $z^{+}=W_{\mathbf{q}}^{(1)}\left(\overline{\mathcal{T}}_{1}\right)=$ $W_{\mathbf{q}}\left(\mathcal{M}_{+}\right)=Z_{\mathbf{q}}^{+}$, and $z^{\diamond}=W^{(2)}\left(\overline{\mathcal{T}}_{2}\right)=Z_{\mathbf{q}}^{\diamond}$, as obtained by displaying, in a similar way,

$$
\frac{W_{\mathbf{q}}^{(2)}(\{\mathbf{t}, e\})}{z^{\diamond}}=\prod_{u \in \mathbf{t}} \bar{\zeta}^{(e(u))}\left(\mathbf{w}^{\mathbf{t}}(u)\right)=P_{\bar{\zeta}}^{(2)}(\{\mathbf{t}, e\}), \quad \mathbf{t} \in \overline{\mathcal{T}}_{2},
$$

hence the uniqueness of the good solution. Moreover, $Z_{\mathbf{q}}=2 Z_{\mathbf{q}}^{+}-1+\left(Z_{\mathbf{q}}^{\diamond}\right)^{2}$ is finite, hence $\mathbf{q}$ is admissible.

\subsection{Proof of Theorem 1}

In order to complete the proof of Theorem 1 , and since we know thanks to Propositions 3 and 2 that certain metric properties of $P_{\mathbf{q}}$-distributed maps are encoded into SGW trees, we would like to apply Theorem 2 to the measures $\mathbb{P}_{\boldsymbol{\zeta}, \boldsymbol{\nu}^{0}}^{(1)}$ and $\mathbb{P}_{\boldsymbol{\zeta}, \boldsymbol{\nu}^{0}}^{(2)}$ obtained in Proposition 3 However, there is still an obstacle that prevents us from doing this - namely, the spatial displacements associated with $\nu^{0}$ are not all centered.

Fortunately, it holds, as we will see, that these become centered after some mild manipulations, namely, a deterministic translation by $1 / 2$ of the labels of vertices of type 2 , and a random shuffling of the kind of Section 2.4, which does not change the values of the labels, though it does change the planar structure of the tree. In the sequel we discuss only the case of $P_{\mathbf{q}}^{+}$and keep in mind that $P_{\mathbf{q}}^{0}$ can be given a similar treatment, taking into account that we have to manipulate two trees instead of one.

Let $\widetilde{\mathbb{T}}$ be the image of $\overline{\mathbb{T}}$ under the mapping $\psi:(\mathbf{t}, e, \ell) \mapsto\left(\mathbf{t}, e,\left(\ell_{u}+\mathbb{1}_{\{e(u)=2\}} / 2, u \in \mathbf{t}\right)\right)$, so that an element of $\widetilde{\mathbb{T}}$ is obtained from an element of $\overline{\mathbb{T}}$ by adding $1 / 2$ to all the labels of ' $\diamond$ ' (vertices of type 2). We let $\widetilde{\Psi}^{+}=\psi \circ \Psi^{+}$, where $\Psi^{+}$is the bijection of Proposition 2 As an elementary consequence of Proposition 3, for an admissible sequence $\mathbf{q}$, the law of $\widetilde{\Psi}^{+}(M)$ under $P_{\mathbf{q}}^{+}$is $\mathbb{P}_{\boldsymbol{\zeta}, \boldsymbol{\nu}}^{(1)}$, where

- $\zeta$ is described in the statement of Proposition 3 .

- $\nu_{1, \mathbf{w}}=\nu_{1, \mathbf{w}}^{0}$,

- for $\mathbf{w} \in \mathcal{W}_{3}$ with $p_{3}(\mathbf{w})=0, \nu_{2, \mathbf{w}}$ is the image of $\nu_{2, \mathbf{w}}^{0}$ by $\left(y_{j}, 1 \leq j \leq|\mathbf{w}|\right) \mapsto\left(y_{j}-\right.$ $\left.\mathbb{1}_{\left\{w_{j}=1\right\}} / 2,1 \leq j \leq|\mathbf{w}|\right)$, and

- for $\mathbf{w} \in \mathcal{W}_{3}$ with $p_{3}(\mathbf{w})=0, \nu_{3, \mathbf{w}}$ is the image of $\nu_{3, \mathbf{w}}^{0}$ by $\left(y_{j}, 1 \leq j \leq|\mathbf{w}|\right) \mapsto\left(y_{j}+\right.$ $\left.\mathbb{1}_{\left\{w_{j}=2\right\}} / 2,1 \leq j \leq|\mathbf{w}|\right)$.

Lemma 2 The spatial displacements $\overleftrightarrow{\nu}$ defined from $\nu$ as in Lemma 1 are centered and satisfy the moment assumptions of Theorem 2

Proof. It is immediate to check that the measures $\nu_{i, \mathbf{w}}(\mathrm{d} \mathbf{y})$, for $i \in[3]$ and $\mathbf{w} \in \mathcal{W}_{3}$, are supported by $\left\{\mathbf{y}: \max _{1 \leq i \leq|\mathbf{w}|} y_{i} \leq|\mathbf{w}|\right\}$, so that the moment conditions trivially hold. It thus remain to see that $\nu_{i, \mathbf{w}}$ is centered. This is trivial for $i=1$. Let us show it for $i=3$, the case $i=2$ being similar. In this case, $\nu_{3, \mathbf{w}}$ is the law of

$$
\left(\sum_{j=1}^{i} X_{j}+\mathbb{1}_{\left\{w_{i}=2\right\}} / 2,1 \leq i \leq|\mathbf{w}|\right),
$$

where $\left(X_{j}+\mathbb{1}_{\left\{w_{j-1}=1\right\}}, 1 \leq j \leq|\mathbf{w}|+1\right)$ (with $w_{0}=1$ ) is uniform on the set of non-negative sequences with $|\mathbf{w}|+1$ terms and total sum $p_{1}(\mathbf{w})+1$. As a consequence, this last sequence is exchangeable and we deduce that, whenever $p(\mathbf{w})=\left(k, k^{\prime}, 0\right)$,

$$
E\left[X_{j}\right]=\frac{k+1}{k+k^{\prime}+1}-\mathbb{1}_{\left\{w_{j-1}=1\right\}} \quad 1 \leq j \leq k+k^{\prime} .
$$

Next, under $\nu_{3, \overleftarrow{\mathbf{w}}}$, a similar formula hold, replacing the indicator by $\mathbb{1}_{\left\{w_{|\mathbf{w}|-j+2}=1\right\}}$, so that $\overleftarrow{\nu}_{3, \overleftarrow{\mathbf{w}}}$ is the law of $\left(\sum_{j=1}^{k+k^{\prime}-i+1} X_{j}+\mathbb{1}_{\left\{w_{i}=2\right\}} / 2,1 \leq i \leq|\mathbf{w}|\right)$ where this time $\left(X_{j}+\mathbb{1}_{\left\{w_{k+k^{\prime}-j+2}=1\right\}}, 1 \leq j \leq|\mathbf{w}|+1\right)$ 
(with $w_{k+k^{\prime}+1}=1$ ) is uniform on the set (17). Thus, using (23), and since there are $k+2$ terms equal to 1 in the sequence $w_{0}, w_{1}, \ldots, w_{k+k^{\prime}+1}$,

$$
\begin{aligned}
\frac{\nu_{3, \mathbf{w}}\left(y_{i}\right)+\overleftarrow{\nu}_{3, \overleftarrow{\mathbf{w}}}\left(y_{i}\right)}{2} & =\frac{\mathbb{1}_{\left\{w_{i}=2\right\}}}{2}+\frac{1}{2}\left(k+1-\sum_{j=0}^{i-1} \mathbb{1}_{\left\{w_{j}=1\right\}}-\sum_{j=i+1}^{k+k^{\prime}+1} \mathbb{1}_{\left\{w_{j}=1\right\}}\right) \\
& =\frac{1}{2}\left(\mathbb{1}_{\left\{w_{i}=2\right\}}+k+1-\left(k+1+1-\mathbb{1}_{\left\{w_{i}=1\right\}}\right)\right)=0 .
\end{aligned}
$$

Hence the result.

Proof of Theorem 1] From Proposition 2, it holds that $\mathcal{R}(M)$ under $P_{\mathbf{q}}^{+}(\cdot \mid \# V(M)=n)$ has same distribution as $\max _{u \in T^{(1)}} L_{u}-\min _{u \in T^{(1)}} L_{u}+1$ under $\mathbb{P}_{\boldsymbol{\zeta}, \boldsymbol{\nu}^{0}}^{(1)}\left(\cdot \mid \# T^{(1)}=n-1\right)$. Since the mapping $\psi: \overline{\mathbb{T}} \rightarrow \widetilde{\mathbb{T}}$ defined above does not affect the labels of vertices of type 1 , the same statement holds where $\mathbb{P}_{\boldsymbol{\zeta}, \boldsymbol{\nu}}^{(1)}$ replaces $\mathbb{P}_{\boldsymbol{\zeta}, \boldsymbol{\nu}^{0}}^{(1)}$, or even if we take $\mathbb{P}_{\boldsymbol{\zeta}, \overleftrightarrow{\boldsymbol{\nu}}}^{(1)}$ instead, since shuffling does not affect the (multi)sets $\left\{\ell_{u}, u \in \mathbf{t}\right\}$. Moreover, it is elementary to check from the labeling constraints 5. (a,b) in Section 3.1, that a.s. under $\mathbb{P}_{\boldsymbol{\zeta}, \overleftrightarrow{\nu}}^{(1)}$

$$
0 \leq\left(\max _{u \in T} L_{u}-\min _{u \in T} L_{u}+1\right)-\left(\max _{u \in T^{(1)}} L_{u}-\min _{u \in T^{(1)}} L_{u}+1\right) \leq 1 / 2 .
$$

Now, by Theorem 2, under $\mathbb{P}_{\zeta, \overleftrightarrow{\nu}}^{(1)}\left(\cdot \mid \# T^{(1)}=n-1\right)$, it holds that $n^{-1 / 4}(\max L-\min L)$ converges in distribution to $C_{\mathbf{q}}\left(\max R^{\mathrm{ex}}-\min R^{\mathrm{ex}}\right)$, which combined with 24 yields the wanted result, where $C_{\mathbf{q}}=\Sigma \sqrt{2 /\left(\sigma \sqrt{a_{1}}\right)}$ is entirely determined by $\mathbf{q}$ via $\boldsymbol{\mu}$ and $\boldsymbol{\nu}^{0}$.

The assertions (ii) and (iii) are now obtained following the same lines as (Marckert and Miermont, 2006 Sect. 3.3), and we only sketch the arguments. For (ii), Proposition 2 entails that under $P_{\mathbf{q}}^{+}$, the distance $d_{M}\left(r, r^{\prime}\right)$, for $r^{\prime}$ picked uniformly at random in $V(M) \backslash\{r\}$, has same distribution as $L_{u^{\prime}}-\min _{T^{(1)}} L+1$ under $\mathbb{P}_{\boldsymbol{\zeta}, \overleftrightarrow{\nu}}^{(1)}$, where $u^{\prime}$ is picked uniformly at random in $T^{(1)}$ (this involves an enlargement of the probability space, which we omit for simplicity). Similarly as above,

$$
\left|\left(L_{u^{\prime}}-\min _{T^{(1)}} L+1\right)-\left(L_{u^{\prime}}-\min L\right)\right| \leq 2,
$$

and recalling the definition of the functions $\Lambda_{i}^{\mathbf{t}}$ given in the statement of Theorem 2, $L_{u^{\prime}}-\min L$ has the same distribution as $S_{\left(\Lambda_{1}^{T}\right)^{-1}\left(\left[\# T^{(1)} U\right]\right)}^{T}-\inf S^{T}$ under $\mathbb{P}_{\boldsymbol{\zeta}, \overleftrightarrow{\boldsymbol{\nu}}}^{(1)} \otimes \mathrm{d} u \mathbb{1}_{[0,1]}$, so that $U(u)=u$ is uniform on $[0,1]$ and independent of $T$. Conditioning on $\# T^{(1)}=n-1$, we obtain the result from the convergence of $n^{-1 / 4} S_{[\# T \cdot]}^{T}$ and the fact that $(\# T)^{-1} \Lambda_{1}^{T}([\# T \cdot])$ converges to $\left(a_{1} t, 0 \leq t \leq 1\right)$, so that $(\# T)^{-1}\left(\Lambda_{1}^{T}\right)^{-1}\left(\left[\# T^{(1)}.\right]\right)$ converges to the identity of $[0,1]$. This allows to conclude that under $P_{\mathbf{q}}^{+}(\cdot \mid \# V(M)=n), n^{-1 / 4} d_{M}\left(r, r^{\prime}\right)$ converges in distribution to $C_{\mathbf{q}}\left(R_{U}^{\mathrm{ex}}-\inf R^{\mathrm{ex}}\right)$ where $U$ is uniform on $[0,1]$ and independent of $R^{\mathrm{ex}}$, and this has same distribution as $C_{\mathbf{q}} \sup R^{\mathrm{ex}}$ by the properties of symmetry and invariance under uniform re-rooting of the Brownian snake that can be found in Le Gall and Weill (2006).

For (iii), we observe from $\sqrt{14}$ that for every bounded Lipschitz $g: \mathbb{R}_{+} \rightarrow \mathbb{R}, \mathcal{J}_{n}^{M}(g)$ under $P_{\mathbf{q}}^{+}$has same distribution as

$$
\frac{\# T^{(1)}}{1+\# T^{(1)}} \int_{0}^{1} g\left(\frac{S_{[\# T t]}^{T}-\inf S^{T}+1}{n^{1 / 4}}\right) \frac{\mathrm{d} \Lambda_{1}^{T}([\# T t])}{\# T^{(1)}}+\frac{g(0)}{\# T^{(1)}+1}
$$

under $\mathbb{P}_{\boldsymbol{\zeta}, \overleftrightarrow{\boldsymbol{\nu}}}^{(1)}$. Conditioning on $\# T^{(1)}=n-1$ and using Theorem 2 allows to conclude that Theorem 1 holds for $P_{\mathbf{q}}^{+}$-distributed random maps instead of $P_{\mathbf{q}}$.

Since the study of $P_{\mathbf{q}}^{0}$ involves two trees when applying $\Psi^{0}$, it might look at this point that things do not go as smoothly as in the above case. However, we claim that as $n \rightarrow \infty$, when conditioning two independent multitype Galton-Watson trees to have $n-1$ individuals of type 1 , one of the two trees asymptotically vanishes when compared to the other, so that one might as well deal with a single conditioned tree. This is quite standard and comes from the fact that the law of the total population in a Galton-Watson tree whose offspring distribution has finite variance is in the domain of attraction of a stable law with index $1 / 2$. We refer to Miermont (2006) for details on this. Since we condition on the number of vertices of type 1 as above, the scaling constant $C_{\mathbf{q}}$ is the same, hence the result. 


\subsection{More on the scaling constant $C_{\mathbf{q}}$}

We finish this section by adding a few more details on how the scaling constant $C_{\mathbf{q}}$ can be computed. Since the quantities $\mathbf{a}, \mathbf{b}, \mathbf{Q}, \sigma$ appearing in Section 2.3 can be computed from $\mathbf{q}$ via the functions $f_{\mathbf{q}}^{\bullet}, f_{\mathbf{q}}^{\diamond}$, it remains to compute the variances of the spatial displacements.

\subsubsection{Variances of the spatial displacements}

We thus compute the variances $\overleftrightarrow{\nu}_{i, \mathbf{w}}\left(y_{j}^{2}\right)$ that are involved in the computation of $\Sigma$, as defined in 11 , when $\boldsymbol{\nu}$ is the spatial displacement defined from $\boldsymbol{\nu}^{0}$ as above. Note that if $X_{1}, \ldots, X_{n}$ are uniform in $\left\{\left(k_{1}, \ldots, k_{n}\right) \in \mathbb{Z}_{+}^{n}: k_{1}+\ldots+k_{n}=m\right\}$, which has $\left(\begin{array}{c}n+m-1 \\ n-1\end{array}\right)$ elements, then $X_{1}, \ldots, X_{n}$ are exchangeable, and the law of $X_{1}$ is a mixture of binomial distributions, namely, if $\vartheta$ is a random variable in $[0,1]$ with $P(\vartheta \in \mathrm{d} \theta)=(n-1)(1-\theta)^{n-2} \mathbb{1}_{[0,1]}(\theta) \mathrm{d} \theta$, then $X_{1}$ follows a $\mathcal{B}(m, \vartheta)$ distribution (i.e. $\mathcal{B}(m, \theta)$ given $\vartheta=\theta$ ). Elementary computations show that

$$
\operatorname{Var} X_{j}=\frac{m(n-1)(n+m)}{n^{2}(n+1)} .
$$

By exchangeability, $\operatorname{Var}\left(X_{1}+\ldots+X_{i}\right)=i \operatorname{Var} X_{1}+i(i-1) \operatorname{Cov}\left(X_{1}, X_{2}\right)$, and this is 0 for $i=n$ since $X_{1}+\ldots+X_{n}=m$. Thus, $\operatorname{Cov}\left(X_{1}, X_{2}\right)=-\operatorname{Var} X_{1} /(n-1)$, and we find that

$$
\operatorname{Var}\left(X_{1}+\ldots+X_{i}\right)=\frac{i(n-i) m(n+m)}{n^{2}(n+1)} .
$$

From this, one can check that

$$
\begin{aligned}
& \overleftrightarrow{\nu}_{3, \mathbf{w}}\left(y_{j}^{2}\right)=\frac{j\left(k+k^{\prime}+1-j\right)(k+1)\left(2 k+k^{\prime}+2\right)}{\left(k+k^{\prime}+1\right)^{2}\left(k+k^{\prime}+2\right)}, \\
& \overleftrightarrow{\nu}_{2, \mathbf{w}}\left(y_{j}^{2}\right)=\frac{j\left(k+k^{\prime}+1-j\right) k\left(2 k+k^{\prime}+1\right)}{\left(k+k^{\prime}+1\right)^{2}\left(k+k^{\prime}+2\right)},
\end{aligned}
$$

whenever $p(\mathbf{w})=\left(k, k^{\prime}, 0\right)$.

\subsubsection{Back to triangulations}

We finish by computing the scaling constant $C_{\mathbf{q}}$ in the case of triangulations, that we began to study in Section 1.3 Recall the critical values for $q=q_{3}, Z_{\mathbf{q}}^{+}, Z_{\mathbf{q}}^{\diamond}$ computed there. In this case, $\mu^{(1)}(0,0, k)=(1-$ $1 / \sqrt{3})^{k} / \sqrt{3}$, which corresponds to a geometric law with mean $\sqrt{3}-1$ and variance $3-\sqrt{3}, \mu^{(2)}(1,0,0)=$ $(3+\sqrt{3}) / 6, \mu^{(2)}(0,2,0)=(3-\sqrt{3}) / 6$, and $\mu^{(3)}(0,1,0)=1$. This gives

$$
\mathfrak{M}_{q_{\text {cr }} \delta_{3}}=\left(\begin{array}{ccc}
0 & 0 & \sqrt{3}-1 \\
\frac{3+\sqrt{3}}{6} & \frac{3-\sqrt{3}}{3} & 0 \\
0 & 1 & 0
\end{array}\right),
$$

which admits for left and right eigenvectors

$$
\mathbf{a}=\frac{1}{3}(1,3-\sqrt{3}, \sqrt{3}-1), \quad \mathbf{b}=\frac{3(\sqrt{3}-1)}{2}(\sqrt{3}-1,1,1),
$$

normalized according to the convention of Section 2.3 . The associated matrices $\mathbf{Q}$ are then

$$
Q^{(1)}=\left(\begin{array}{ccc}
0 & 0 & 0 \\
0 & 0 & 0 \\
0 & 0 & 2(\sqrt{3}-1)^{2}
\end{array}\right), \quad Q^{(2)}=\left(\begin{array}{ccc}
0 & 0 & 0 \\
0 & \frac{3-\sqrt{3}}{3} & 0 \\
0 & 0 & 0
\end{array}\right), \quad Q^{(3)}=0 .
$$

We find $\sigma^{2}=9(7-4 \sqrt{3})=0.64617 \ldots$, and $\Sigma^{2}=(2-\sqrt{3}) / 2=0.13397 \ldots$ This allows to find formula (8).

\section{Miscellaneous comments}

1. We stress that it is possible to adapt the methods of the present paper to treat the most general case of random Eulerian (face-bicolourable, say black and white) planar maps with a Boltzmann distribution, with 
energy $\log q_{k}^{\circ}$ per white face of degree $k$, and $\log q_{k}^{\bullet}$ per black face of degree $k$. Under the assumption that e.g. the black faces have uniformly bounded degree, it is possible to use the most elaborate bijection of Bouttier et al. (2004), to obtain a correspondence between such Boltzmann distributions and laws of $K$-type SGW trees, where the number $K$ of types needed depends on the bound on the degree of black faces.

2. It is likely that methods similar to that of Le Gall (2006) would allow, starting from Theorem 1 , to obtain a similar result for Boltzmann distributions on the set $\overrightarrow{\mathcal{M}}$ of rooted maps, where this time, the base vertex is taken to be the source of the root edge. These distributions are obtained from our model by conditioning the source of $\vec{e}$ to be the distinguished vertex $e_{-}=r$, and this amounts to condition the labels of the associated SGW tree of Proposition 3 to be all non-negative. We plan to pursue this in future work.

3. It would also be of interest to condition the map not to have loops, i.e. to impose extra connectivity constraints, as mentioned in Section 1.3. In this respect, we mention that, according to the description of the BDFG bijection in Bouttier et al. (2004), conditioning a $P_{\mathbf{q}}^{+}$-distributed map not to have any loops is the same as the following: re-root the typical spatial tree $(\mathbf{t}, e, \ell)$ under $\Psi_{*}^{+} P_{\mathbf{q}}^{+}$at any of the type- 1 vertices of minimal label (call the re-rooted tree $\mathbf{t}_{*}$, and let $\ell^{*}$ be the associated labeling function), and condition on the event that every $u \in \mathbf{t}_{*}^{(2)}$, there exists at least one strict descendent $v \in \mathbf{t}_{*}^{(1)}$ of $u$, with $\ell_{v}^{*}=\ell_{u}^{*}$.

4. The methods used to prove Theorem 1 should also entail convergence of the rescaled random maps under consideration to the Brownian map defined in Marckert and Mokkadem (2006), in a sense close to that discussed in the latter article. The Brownian map is a conjectured limit of renormalized conditioned random maps of the form that are considered in the present article, where the word 'limit' should holds in an appropriate space of (isometry classes of) metric spaces, e.g. the space of compact metric spaces endowed with the Gromov-Hausdorff distance, see Burago et al. (2001). The results of Le Gall (2006) could probably be generalized to our context as well.

\section{References}

J. Ambjørn, B. Durhuus, and T. Jonsson. Quantum geometry. A statistical field theory approach. Cambridge Monographs on Mathematical Physics. Cambridge University Press, Cambridge, 1997. ISBN 0-52146167-7.

O. Angel. Growth and percolation on the uniform infinite planar triangulation. Geom. Funct. Anal., 13(5): 935-974, 2003. ISSN 1016-443X.

J. Bouttier, P. Di Francesco, and E. Guitter. Planar maps as labeled mobiles. Electron. J. Combin., 11: Research Paper 69, 27 pp. (electronic), 2004. ISSN 1077-8926.

D. Burago, Y. Burago, and S. Ivanov. A course in metric geometry, volume 33 of Graduate Studies in Mathematics. American Mathematical Society, Providence, RI, 2001. ISBN 0-8218-2129-6.

P. Chassaing and B. Durhuus. Local limit of labelled trees and expected volume growth in a random quadrangulation. Ann. Probab., 34(3):879-917, 2006.

P. Chassaing and G. Schaeffer. Random planar lattices and integrated superBrownian excursion. Probab. Theory Related Fields, 128(2):161-212, 2004. ISSN 0178-8051.

R. Cori and B. Vauquelin. Planar maps are well labeled trees. Canad. J. Math., 33(5):1023-1042, 1981. ISSN 0008-414X.

J.-F. Le Gall. A conditional limit theorem for tree-indexed random walks. Stoch. Process. Appl., 116(4): 539-567, 2006. ISSN 0304-4149.

J.-F. Le Gall. Spatial branching processes, random snakes and partial differential equations. Lectures in Mathematics ETH Zürich. Birkhäuser Verlag, Basel, 1999. ISBN 3-7643-6126-3.

J.-F. Le Gall and M. Weill. Conditioned Brownian trees. Ann. Inst. Henri Poincaré, 2006. To appear.

J.-F. Le Gall. The topological structure of scaling limits of large planar maps. Preprint, 2006.

J.-F. Marckert and G. Miermont. Invariance principles for random bipartite planar maps. Ann. Probab., 2006. To appear. 
J.-F. Marckert and A. Mokkadem. Limit of normalized random quadrangulations: the Brownian map. Ann. Probab., 2006. To appear.

J.-F. Marckert and A. Mokkadem. States spaces of the snake and its tour-convergence of the discrete snake. J. Theoret. Probab., 16(4):1015-1046 (2004), 2003. ISSN 0894-9840.

G. Miermont. The limiting genealogy of a critical multitype Galton-Watson process. In preparation, 2006.

D. Poulalhon. Problèmes énumératifs autour des cartes combinatoires et des factorisations dans le groupe symétrique. PhD thesis, École Polytechnique de Paris, 2002.

D. Poulalhon and G. Schaeffer. A bijection for triangulations of a polygon with interior points and multiple edges. Theoret. Comput. Sci., 307(2):385-401, 2003. ISSN 0304-3975.

G. Schaeffer. Conjugaison d'arbres et cartes combinatoires aléatoires. PhD thesis, Université Bordeaux I, 1998.

V. A. Vatutin and E. E. Dyakonova. The survival probability of a critical multitype Galton-Watson branching process. In Proceedings of the Seminar on Stability Problems for Stochastic Models, Part II (Naleczow, 1999), volume 106, pages 2752-2759, 2001.

M. Weill. Regenerative real trees. 2005. Preprint. 
